\title{
Early medieval large glass beads from Poland: utilitarian and social functions
}

\author{
Velké skleněné korále $v$ raně středověkém Polsku: \\ užitková a sociální funkce
}

\author{
Aleksandra Pankiewicz - Sylwia Siemianowska
}

\begin{abstract}
The study focuses on the early medieval large glass beads from the area of Poland, i.e. specimens whose diameter equals at least $1.5 \mathrm{~cm}$ but usually ca. $2 \mathrm{~cm}$ or more. The main aim of this study is to define their function, considering precise context of discovery of particular specimens, metric data and microscopic analyses. Another important task of the study is to determine whether large beads were local products or imports, and from which region and in what social circumstances they reached the studied area. Alongside macroscopic and stylistic features, chemical composition of glass that was used for production of the beads can be conclusive in this situation. In our opinion, it is a special category of finds that appeared in this part of Europe during the time of cultural and political transformation in the $11^{\text {th }}-13^{\text {th }}$ centuries.
\end{abstract}

glass beads - chemical composition - microscopic analysis - Early Middle Ages - Poland - social changes

Předmětem studie jsou polské nálezy raně středověkých velkých skleněných korálů, tj. exemplářů, jejichž, průměr činí nejméně 1,5 cm, ale obvykle kolem $2 \mathrm{~cm}$ či více. Hlavním cílem je prispět k objasnění jejich funkce na základě nálezového kontextu, metriky a výsledků mikroskopické analýzy. Neméně důležité jsou otázky, zda se jedná o místní produkci či o importy, jaká je jejich geografická distribuce a sociální charakteristika prostředí, ve kterém byly nalezeny. Kromě makroskopicky patrných a morfologických znakü autorky hodnotí též chemické složení korálů. Docházejí k závěru, že velké korály reprezentují samostatnou kategorii nálezů a jejich výskyt odráží kulturní a politické proměny středoevropské společnosti v 11.-13. století.

skleněné korále - chemické složení - mikroskopická analýza - raný středověk - Polsko - společenské změny

\section{Introduction}

Among different forms of glass beads used in the Early Middle Ages in the area of Poland there is a specific category that draws special attention - massive beads, i.e. glass objects in the form of a bead whose outer diameter equals at least $1.5 \mathrm{~cm}$, yet usually reaches ca. $2 \mathrm{~cm}$ or more (tab. 1 ; fig. 1 and 2). Large beads, as unusual artefacts, already caught the attention of the scholars, however, they were mostly just briefly mentioned. Only in few works from the 1970s they were more thoroughly described (e.g. Dekówna 1970; Haevernick $1972^{1}$ ). The finds known at that time were catalogued and their provenance and function were discussed.

The reason to undertake studies on large beads again are discoveries (both during the excavations and in storage rooms in museums) new specimens as well as the possibility of looking at the items from a broader perspective. Since the mentioned works were published, the basis for comparative studies has considerably grown, including local glass

${ }^{1}$ This work refers to the Roman period, therefore it is useful for this study only to a minor degree. 
artefacts and those from other parts of Europe, the Middle East, Central Asia and Northern Africa. The presence of new finds of large beads from the area of Poland has been already signaled (Pankiewicz - Siemianowska - Sadowski 2017, 44, cat. no. F1-5), but the problem of their function was just succinctly mentioned. Nevertheless, the way they were used remains unexplained. They are usually interpreted as glass decorations of considerable size elements of necklaces or glass knobs. Just as often they are regarded as spindle whorls, and less frequently as e.g. amulets or game pieces (Krumphanzlová 1965, 164; Dekówna 1970, 38-43; Haevernick 1972; Steppuhn 1998, 75-76; Pankiewicz - Siemianowska Sadowski 2017, 44; Procházka 2018, 159).

The aim of the paper is an attempt to determine the real function of these objects and presenting the unusual context in which they occurred, as the emergence of those large glass beads is, in the authors' opinion, one of the signs of more significant social changes that can be traced in early medieval Poland.

\section{Material and methods}

In the in-depth analysis of function of large beads the finds from the area of Silesia were taken into consideration - 12 specimens, discovered on Ostrów Tumski (Cathedral Island) and at Nowy Targ (New Market) Square in Wrocław, on Ostrówek in Opole, at the cemeteries in Radzików and Stary Zamek, and at other sites: Czeladź Wielka (Sądowel), Kamień Górowski, Grodków (or vicinity of Grodków), Toszowice and Tworków (fig. $1: a-h ; 2: a$, $e, f-g, j-k ; t a b .1)$. The access to the artefacts enabled metric analysis, identification of chemical composition of glass in the case of some objects and also precise recognition of the context of discovery (in the case of the specimens from Wrocław and Opole). The other finds of large beads from Poland and Europe will be used as a comparative basis for the study on the way the discussed objects were used. Although the paper focuses on early medieval beads, we will refer to the finds from earlier and later periods, because the chronology of some specimens is uncertain and also due to the fact that we are going to look for many different functions they could have served. In the research on beads precise measurements play a very important role. An analysis of size, weight, diameter, shape and the situation of the hole in relation to the object's axis can have a key meaning for the attempt at explaining how they were used.

Defining the composition of glass which was used to produce the beads make it possible to determine whether they were local products or imports and to what extent they can be perceived as luxury goods. Fully preserved items were examined using a scanning electron microscope with EDS, which enables an elemental analysis of chemical composition of the observed objects. The analysis was performed using JEOL JSM-6380LA (Japan) scanning electron microscope merged with an EDS electron microprobe, in the following conditions: Acc Volt (acceleration voltage) - $20 \mathrm{kV}$, LC (electron gun current) - $65 \mathrm{~mA}$, WD (working distance) $-10 \mathrm{~mm}$, time of the chemical composition analysis $-100 \mathrm{sec}$. (live time). The elemental analysis, together with appropriate images acquired in the light of backscattered electrons BEI COMPO, was done using the low vacuum technique (40 Pa). In the case of fragmentary preserved objects full analyses of chemical composition of glass were carried out. They were performed using X-ray fluorescence with CAMECA Sx 100 electron probe micro-analyser (EPMA) ${ }^{2}$.

2 For details on the conditions of analyses and a description of methods, see Pankiewicz - Siemianowska Sadowski 2017, 19-12. 
The next stage of the study was an analysis of microscopic traces that may indicate particular ways those large beads were used. Four out of nine discussed items were analysed. The examination was carried out at $6.3 \times$ to $12 \times$ magnification, using an Olympus SZX9 microscope with a Nikon DS-Si3 camera.

\section{Typological classification of large beads}

Maria Dekówna (1970, 32), studying the beads from the area of Poland years ago, paid attention to their remarkable stylistic homogeneity. However, this category of finds, despite appearances, is quite differentiated. The most popular are those described by the author conical or bi-conical ones, spirally wound around the hole, which makes the surface look corrugated (fig. $1: b ; 2: e, g, h$ ). The same result may be achieved through shaping the products in a mould or with the use of glass-working pliers (Dekówna 1970, 30-32), or by pressing the ornament (Wajda 2019, 225). Their common feature is also similar greenish or bluish colour of glass, defined as blue-green, turquoise or sapphire (tab. 1: no. 1, 4, 5, $10,13,14)$. The finds of that kind from the area of Poland are known from Opole, Czersk, Gdańsk, Ciepłe, Sypniewo and Stary Zamek (fig. 1: b and 2: e, g; tab. 1: no. 2, 10-12, 15). The occurrence of similar glass products covers a vast area and has a wide chronology. They are known since the Roman period to the Late Middle Ages and occur in the Mediterranean region and Middle East as well as in Central Europe and Scandinavia (see Dekówna 1970; Haevernick 1972; see also tab. 1 and Chronology of large beads; Large glass beadslocal or foreign product?).

When it comes to shape - bi-conical - and turquoise colour, the bead from Ostrów Tumski in Wrocław is similar to those described above (fig. 1: c; tab. 1: no. 21). However, it differs in terms of the way the surface had been treated, as it was carefully formed yet there is no characteristic spiral 'corrugation'. Completely different are spherical beads, sometimes slightly flattened from one side. They were made of translucent, intensely yellow or green glass. With their colour they resemble 'ordinary' beads, glass rings with gemstones and band rings, yellow and green (cf. fig. 1: $d$, e and Pankiewicz - SiemianowskaSadowski 2017, cat. no. A1-13, B1-3, 28-48, 53-70). Two specimens (from Opole and Wrocław) are undecorated, which is another feature that made them similar to commonly found glass beads. It is worth mentioning that chemical composition of those products is generally similar, or sometimes even identical. During the study a correlation between colour of glass and its chemical composition has been observed (see further).

Very differentiated, in terms of chronology and style, are flat-spherical beads. They do not form a homogeneous group. Among them, there are specimens both non-decorated (fig. $1: g ; 2: d, f, i, j ; t a b .1:$ no. $7,9,16$ ) and richly ornamented with colourful glass threads and dots (fig. $1: a, f ; 2: a-c, k ; t a b .1:$ no. $1,6,8,10$ ). For their production translucent (greenish - fig. $1: a, g ; 2: a, b, j, k$ and tab. $1:$ no. 3, 6-9, 15, 17, 19, 20) and opaque brown or black glass was used (fig. $1: f, h ; 2: c$ and tab. 1: no. 6, 16, 18).

In this remarkably differentiated group it is possible to distinguish items whose stylistics is in some way repetitive. The first subgroup is formed by non-decorated beads made of light-coloured greenish glass, all in almost the same shade. They are characterized by quite large diameter of the hole (fig. $1: g ; 2: j$ ). The category is represented by the finds from Czeladź Wielka, Toszowice and Łęczyca (tab. 1: no. 3, 9, 15). 
a

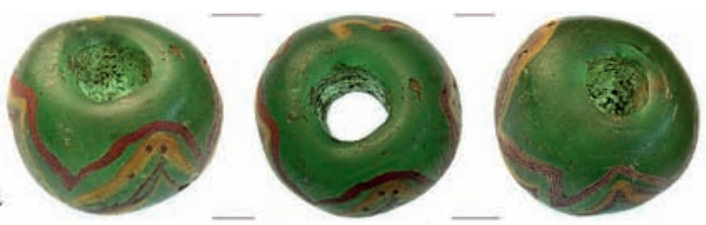

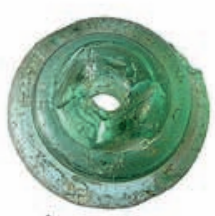

b
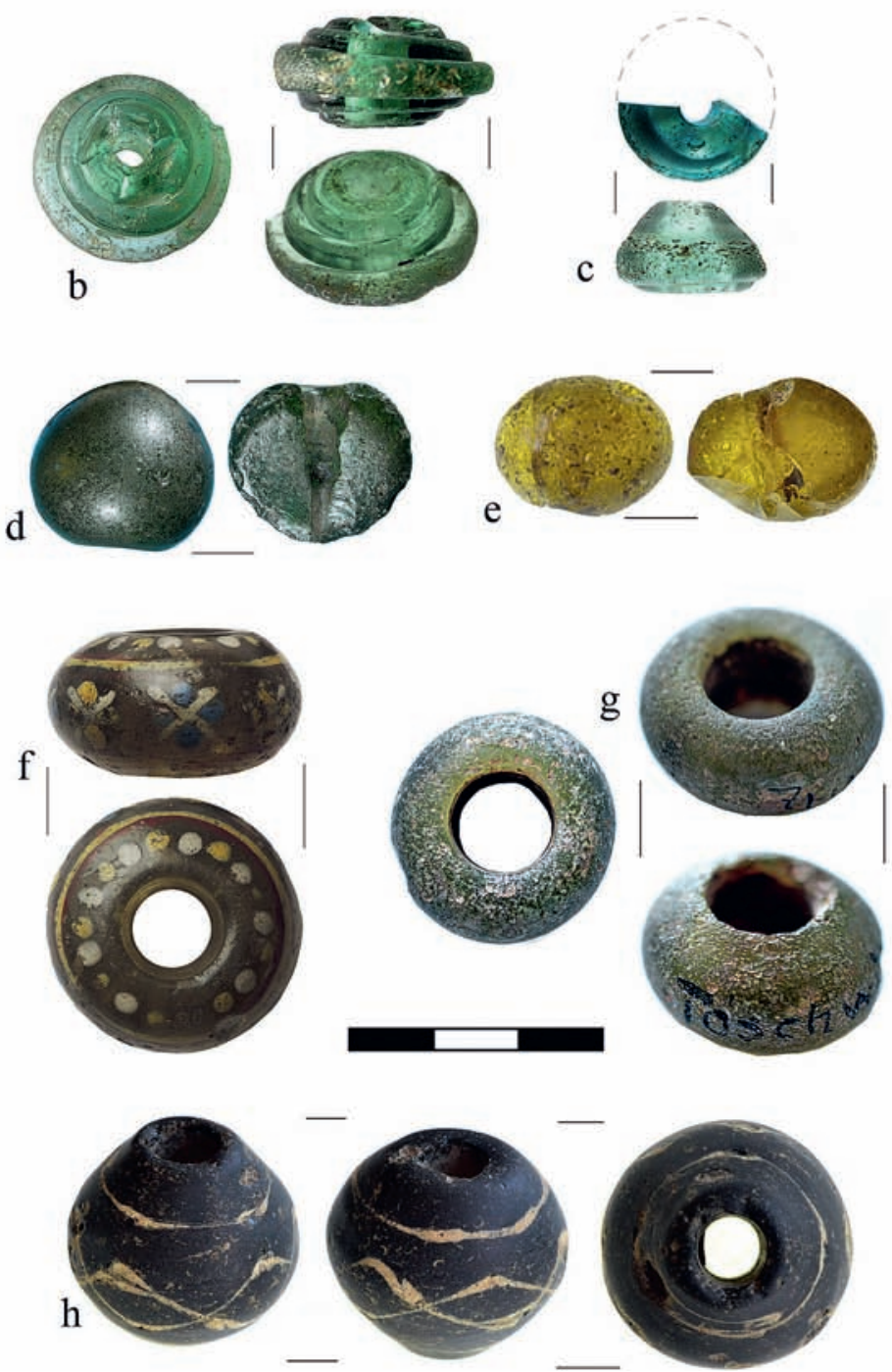

Fig. 1. Large glass beads from Silesia: a/20 - Wrocław Ostrów Tumski, trench I, layer O, plots no. 23-24; b/10 - Opole Ostrówek, layer B1, are 342, m 1e, building no. 18/l; c/21 - Wrocław Ostrów Tumski, trench VII, plot A, layer III; d/11 - Opole-Ostrówek, layer C2, are 311, m 8j, building no. 59; e/22 - Wrocław Ostrów Tumski, trench VI, layer I-III, plot 1200d; f/6 - Grodków or the vicinity of Grodków, context of find unknown; g/15 - Toszowice, Lubin district, context of find unknown; h/16 - Tworków, Racibórz district, site 9 , are $\mathrm{C} 90 \mathrm{a}$, section $\mathrm{C}$, I. Photos by S. Siemianowska. The numbers $(\mathrm{a} / 20, \mathrm{~b} / 10$, etc.) refer to the order numbers in tab. 1 . 

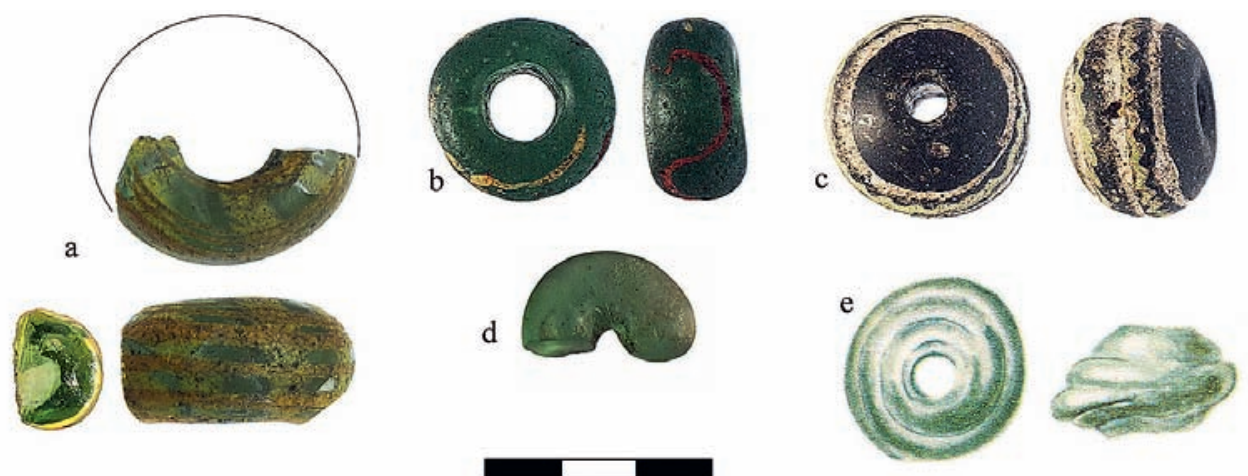

$f$

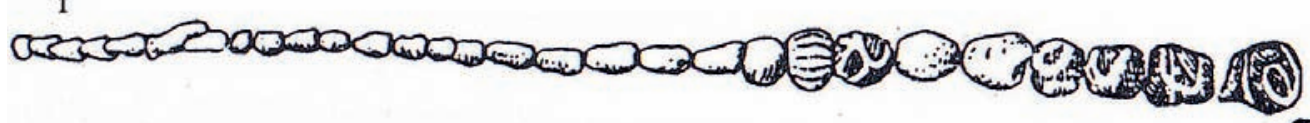

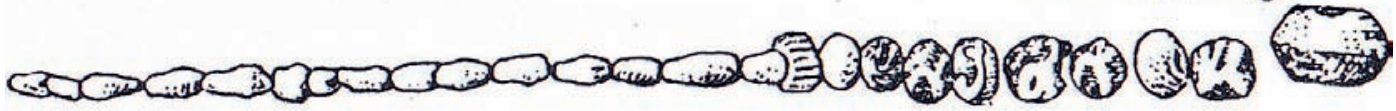
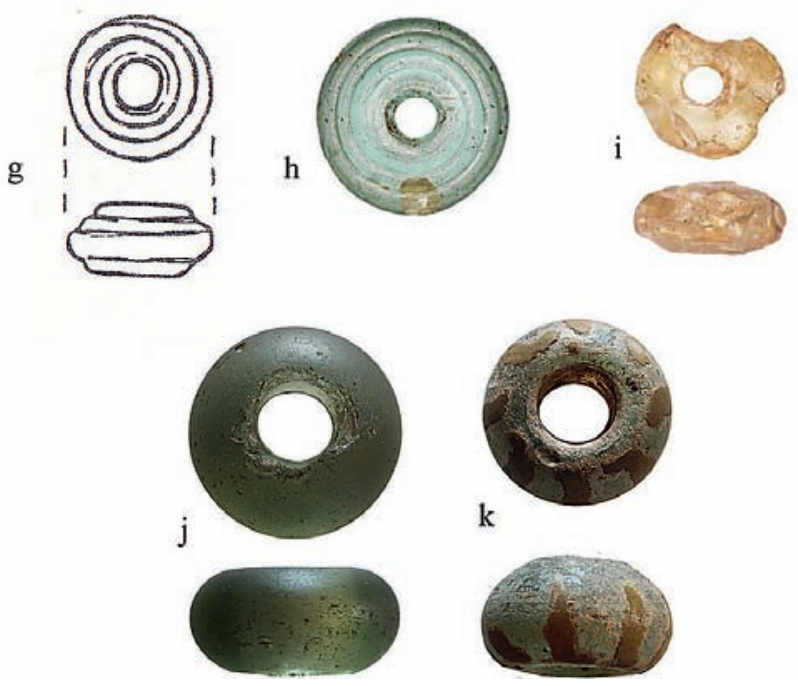

Fig. 2. Large glass beads from Polish lands: a/19 - Wrocław Nowy Targ, layer 19, are 54, quarter C; b/17 Wolin, trench 6/1709, layer XI, after Kokora 2019, fig. 68: 10; c/18 - Wolin trench 6/1660, layer II, after Kokora 2019, fig. 67: 1; d/7 - Janów Pomorski (Truso), topsoil layer, section C, after Dekówna - Purowski 2012, fig. 35/196; e/5 - Gdańsk, $2^{\text {nd }}$ settlement level, street no. 1, after Chmielowska 1960, pl. I: 13; f /12 - Radzików, Dzierżoniów district, grave no. 3, after Wachowski 1975, fig. 20; g/13 - Stary Zamek, Wrocław district, grave no. 70, after Wachowski - Domański 1992, fig. 43; h/1 - Ciepłe, Tczew district, after Wajda 2019, fig. 4.54; i/2 - Ciepłe, Tczew district, after Wajda 2019, fig. 4.54; j/3 - Czeladź Wielka (Sądowel), Góra district, context of find unknown, the cemetery area; k/8 - Kamień Górowski, Góra district, context of find unknown (archival find). a, j, k - photos by A. Pankiewicz. The numbers (a/19, b/17, etc.) refer to the order numbers in tab. 1. 


\begin{tabular}{|c|c|c|c|c|c|c|c|c|c|c|c|c|}
\hline 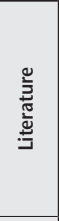 & 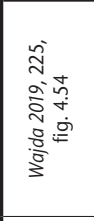 & 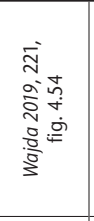 & 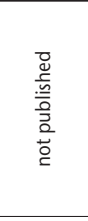 & 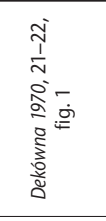 & 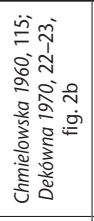 & 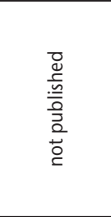 & 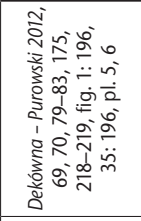 & 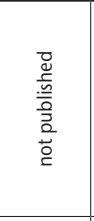 & 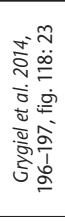 & 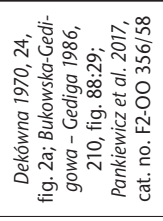 & 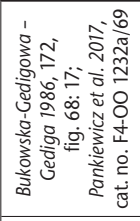 & 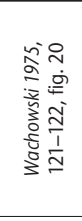 \\
\hline $\begin{array}{l}\text { ते } \\
\text { 잉 } \\
\text { 힌 }\end{array}$ & 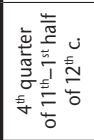 & 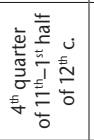 & 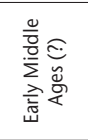 & 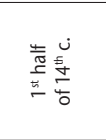 & 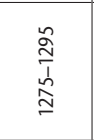 & $\begin{array}{l}\frac{0}{0} \\
\frac{0}{3} \\
\frac{0}{\overline{0}} \\
\frac{0}{2}\end{array}$ & 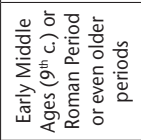 & 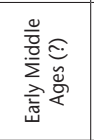 & 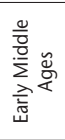 & 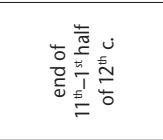 & 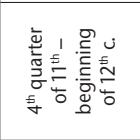 & 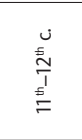 \\
\hline 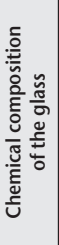 & 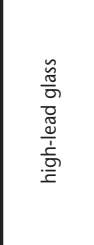 & 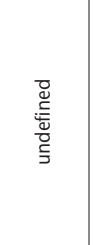 & 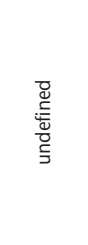 & 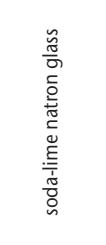 & 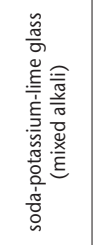 & 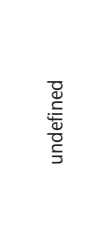 & 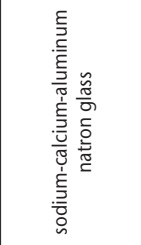 & 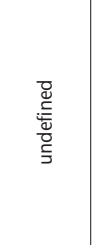 & 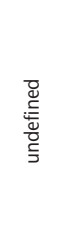 & 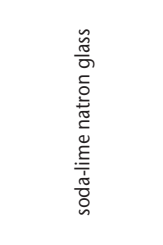 & 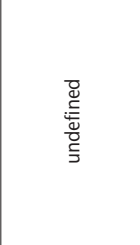 & 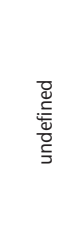 \\
\hline 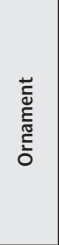 & 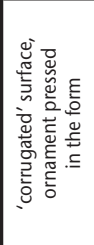 & 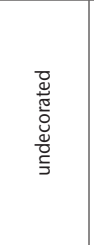 & 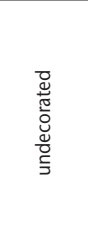 & 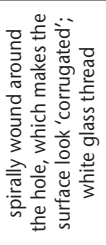 & 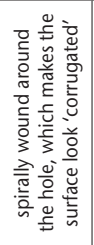 & 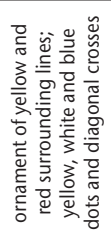 & 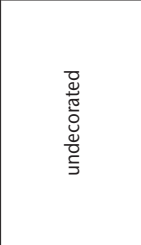 & 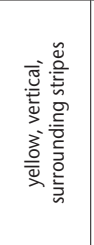 & 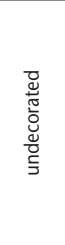 & 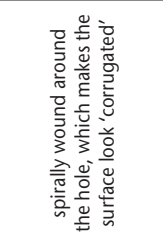 & 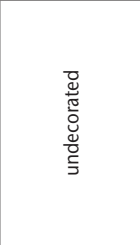 & 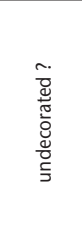 \\
\hline 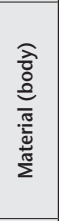 & 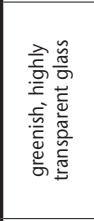 & 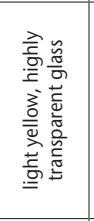 & 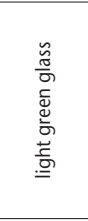 & 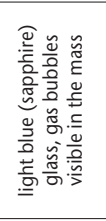 & 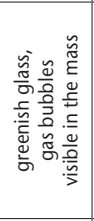 & 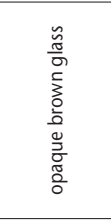 & 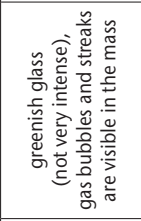 & 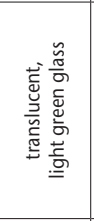 & 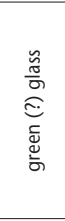 & 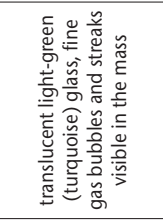 & 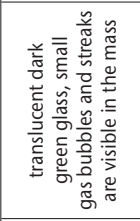 & 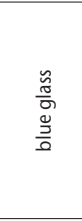 \\
\hline 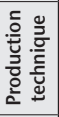 & $\begin{array}{l}\frac{9}{\frac{5}{5}} \\
\frac{5}{3}\end{array}$ & $\begin{array}{l}\text { 亲 } \\
\frac{5}{5} \\
\frac{5}{3}\end{array}$ & 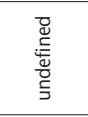 & 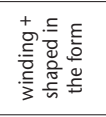 & 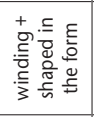 & 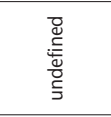 & $\begin{array}{l}\frac{9}{\frac{5}{5}} \\
\frac{5}{3} \\
\frac{5}{3}\end{array}$ & $\begin{array}{l}\frac{0}{\frac{5}{5}} \\
\frac{5}{3} \\
\frac{5}{3}\end{array}$ & 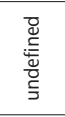 & 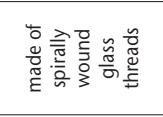 & $\begin{array}{l}\frac{9}{\frac{5}{5}} \\
\frac{5}{5} \\
\frac{5}{3}\end{array}$ & 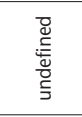 \\
\hline $\begin{array}{l}\text { 离 } \\
\text { 产 } \\
\text { 它 }\end{array}$ & 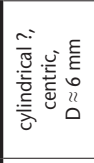 & 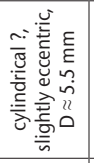 & 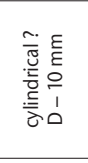 & 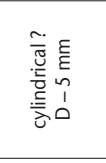 & 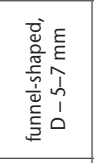 & 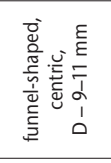 & 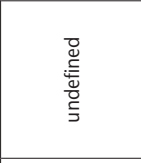 & 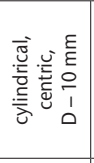 & 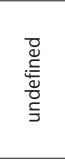 & 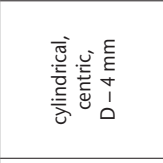 & 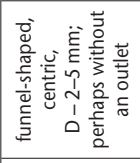 & 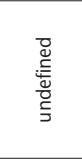 \\
\hline 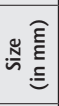 & 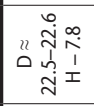 & $\begin{array}{l}\sigma \\
\infty \\
\infty \\
1 \\
1 \\
1 \\
1\end{array}$ & $\begin{array}{l}\stackrel{m}{2}= \\
11 \\
0 \\
0\end{array}$ & 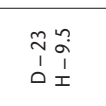 & \begin{tabular}{l}
\multirow{2}{*}{ \pm} \\
1 \\
0 \\
0 \\
1
\end{tabular} & $\begin{array}{ll}0 & 1 \\
1 & 1 \\
0 & 1\end{array}$ & 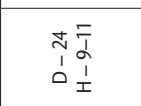 & 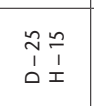 & $\begin{array}{ll}n & \\
& i \\
1 & i \\
0 & 1\end{array}$ & 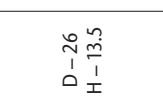 & $\begin{array}{l}\tilde{Z} \bar{T} \\
\dot{1} \\
\end{array}$ & $\begin{array}{l}\frac{\infty}{1} \\
\frac{1}{n} \\
0 \\
0\end{array}$ \\
\hline 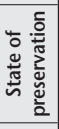 & $\frac{\frac{0}{0}}{\frac{0}{3}}$ & 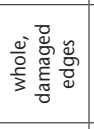 & $\begin{array}{l}\frac{0}{0} \\
\frac{0}{3}\end{array}$ & 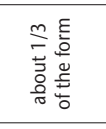 & $\begin{array}{l}\frac{0}{0} \\
\frac{5}{3}\end{array}$ & $\begin{array}{l}\frac{0}{0} \\
\frac{\frac{0}{3}}{3}\end{array}$ & 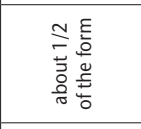 & $\frac{\frac{0}{0}}{\frac{0}{3}}$ & $\frac{\frac{0}{0}}{\frac{0}{3}}$ & $\frac{\frac{0}{0}}{\frac{0}{3}}$ & 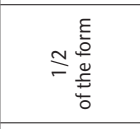 & $\begin{array}{l}\frac{0}{\circ} \\
\frac{\frac{\pi}{3}}{3}\end{array}$ \\
\hline 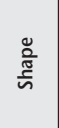 & 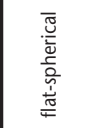 & 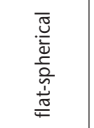 & 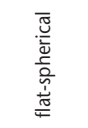 & $\begin{array}{l}\overline{\widetilde{\Xi}} \\
\overline{\tilde{\sigma}}\end{array}$ & 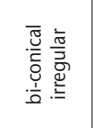 & 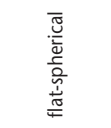 & 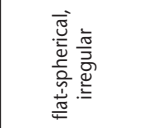 & 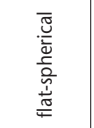 & 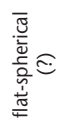 & 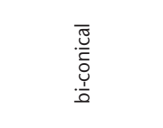 & 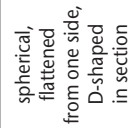 & 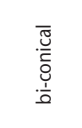 \\
\hline 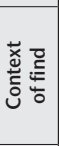 & 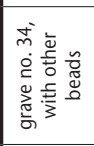 & 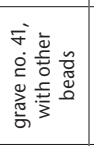 & 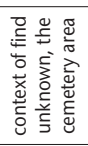 & 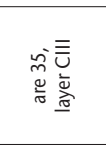 & 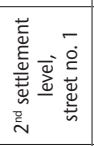 & 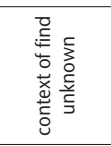 & 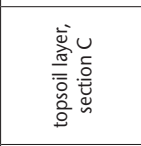 & 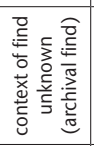 & 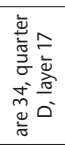 & 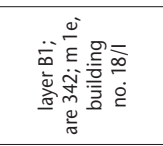 & 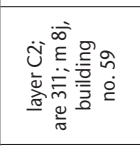 & 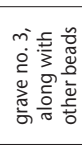 \\
\hline ثँ & 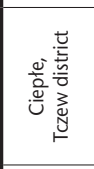 & 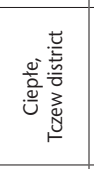 & 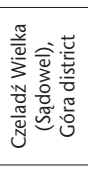 & 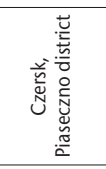 & $\begin{array}{l}\text { 前 } \\
\text { 吾 } \\
0\end{array}$ & 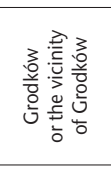 & 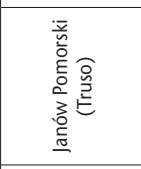 & 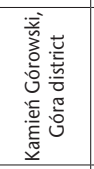 & ฐ్ّ & 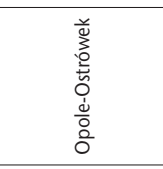 & 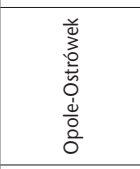 & 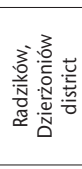 \\
\hline $\begin{array}{l}\dot{\grave{c}} \\
\dot{\grave{I}}\end{array}$ & r. & r. & . & $\frac{\tilde{m}}{0}$ & 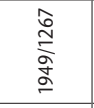 & r. & $\begin{array}{l}\frac{0}{2} \\
\frac{2}{3} \\
\frac{2}{3}\end{array}$ & $\begin{array}{l}\overline{\dot{d}} \\
\text { o̊ }\end{array}$ & 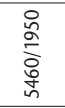 & $\begin{array}{l}\infty \\
\stackrel{\infty}{0} \\
\stackrel{్}{\circ}\end{array}$ & 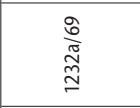 & r. \\
\hline $\bar{j}^{\circ}: \frac{0}{4}$ & $\stackrel{\mathfrak{N}}{\stackrel{N}{S}}$ & $\stackrel{\bar{N}}{\mathrm{~N}}$ & $\stackrel{\bar{d}}{m}$ & $\checkmark$ & $\stackrel{\Xi}{\sim}$ & $\frac{5}{5}$ & $\stackrel{i}{\stackrel{N}{N}}$ & $\underset{\infty}{\stackrel{䒑}{\Delta}}$ & $a$ & $\stackrel{0}{\hat{0}}$ & $\stackrel{\text { OO }}{\equiv}$ & 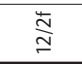 \\
\hline
\end{tabular}




\begin{tabular}{|c|c|c|c|c|c|c|c|c|c|}
\hline 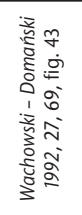 & 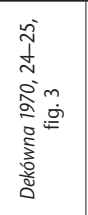 & 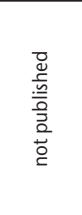 & 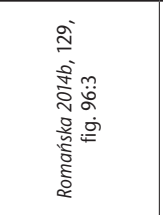 & 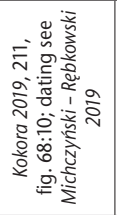 & 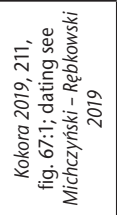 & 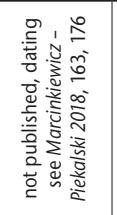 & 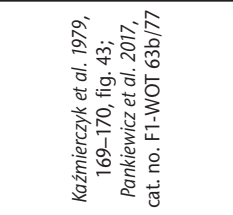 & 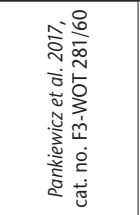 & 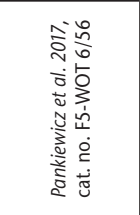 \\
\hline 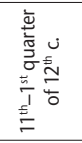 & 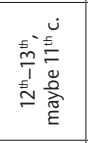 & 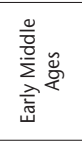 & 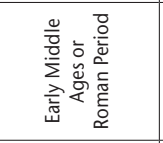 & $\begin{array}{l}8 \\
\frac{0}{1} \\
\frac{2}{2}\end{array}$ & 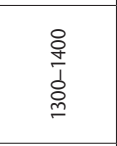 & 岁 & 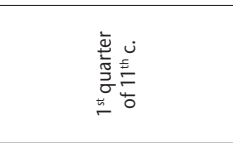 & 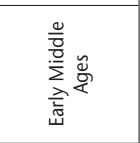 & 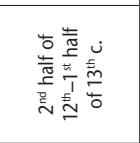 \\
\hline 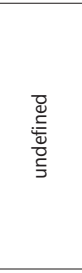 & 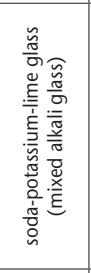 & 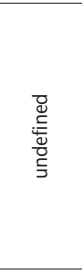 & 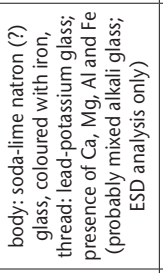 & 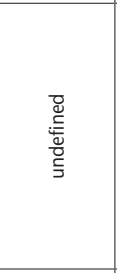 & 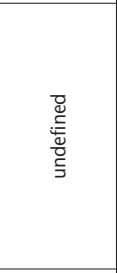 & 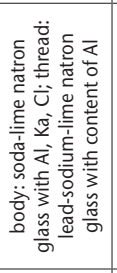 & 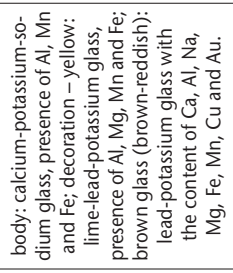 & 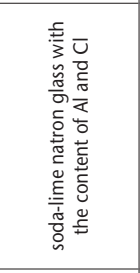 & 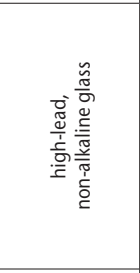 \\
\hline 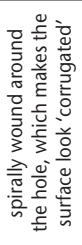 & 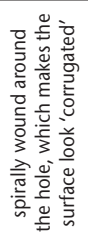 & 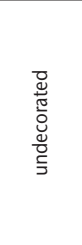 & 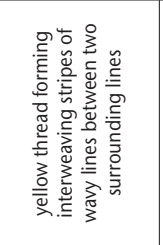 & 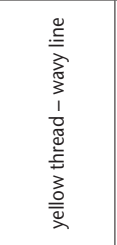 & 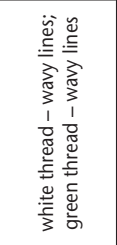 & 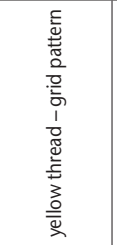 & 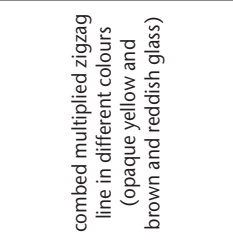 & 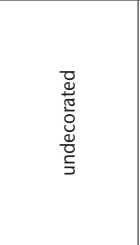 & 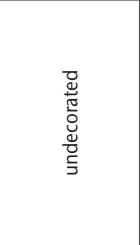 \\
\hline 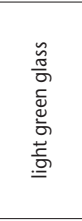 & 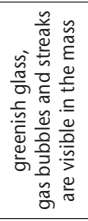 & 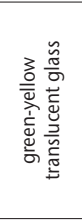 & 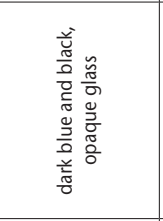 & 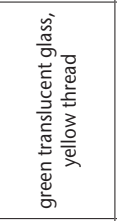 & 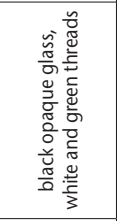 & 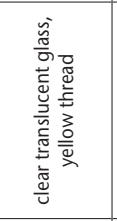 & 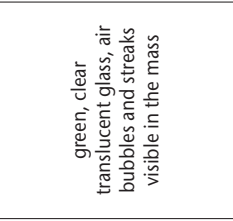 & 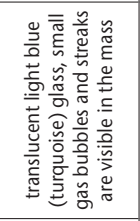 & 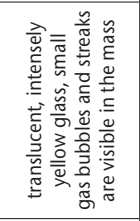 \\
\hline 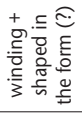 & 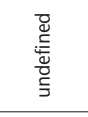 & 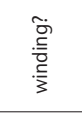 & 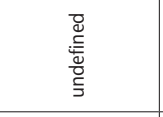 & 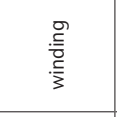 & $\begin{array}{l}\frac{9}{5} \\
\frac{5}{5} \\
\frac{5}{3}\end{array}$ & 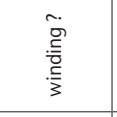 & $\begin{array}{l}\frac{9}{5} \\
\frac{5}{3} \\
\frac{5}{3}\end{array}$ & 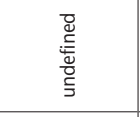 & $\begin{array}{l}\stackrel{0}{5} \\
\stackrel{5}{\frac{5}{3}}\end{array}$ \\
\hline 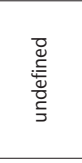 & 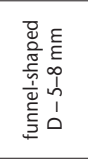 & 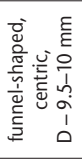 & 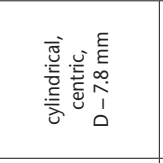 & 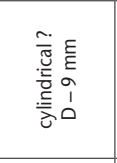 & 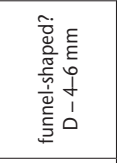 & 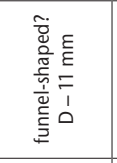 & 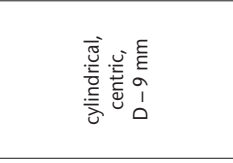 & 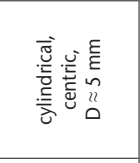 & 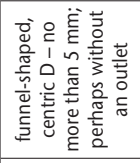 \\
\hline 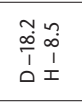 & $\begin{array}{l}\stackrel{5}{1} \\
1 \\
1 \\
0 \\
1 \\
I\end{array}$ & $\begin{array}{l}\underset{n}{\mathbb{N}} \stackrel{?}{=} \\
1 \\
0 \\
1 \\
\pm\end{array}$ & 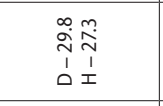 & $\begin{array}{ll}\mathfrak{n} & m \\
1 & \mathfrak{1} \\
1 & 1\end{array}$ & 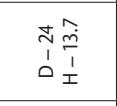 & 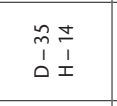 & $\begin{array}{ll}2 & 0 \\
1 & 0 \\
1 & 1 \\
0 & x\end{array}$ & $\begin{array}{l}0 \\
0 \\
1 \\
1 \\
0 \\
1\end{array}$ & \begin{tabular}{l}
$\bar{n}$ \\
$\overline{1}$ \\
1 \\
0 \\
0 \\
\multicolumn{1}{c}{}
\end{tabular} \\
\hline$\frac{\frac{0}{0}}{\frac{0}{3}}$ & $\frac{\frac{0}{0}}{\frac{0}{3}}$ & $\begin{array}{l}\frac{0}{0} \\
\frac{0}{3}\end{array}$ & $\frac{\frac{0}{0}}{\frac{0}{3}}$ & $\frac{\frac{0}{0}}{\frac{0}{3}}$ & $\begin{array}{l}\frac{0}{0} \\
\frac{0}{3}\end{array}$ & 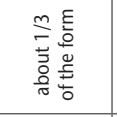 & $\frac{\frac{0}{0}}{\frac{0}{3}}$ & 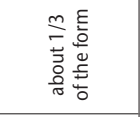 & 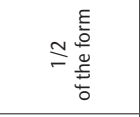 \\
\hline 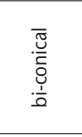 & 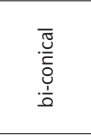 & 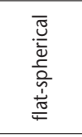 & 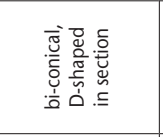 & 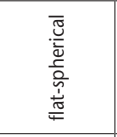 & 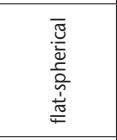 & 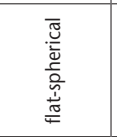 & 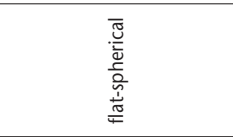 & 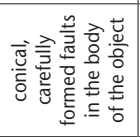 & 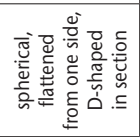 \\
\hline 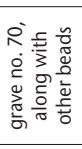 & 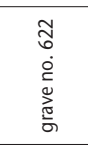 & 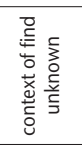 & 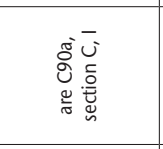 & 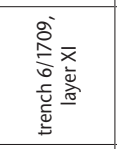 & 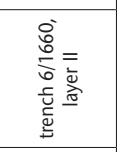 & 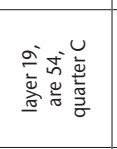 & 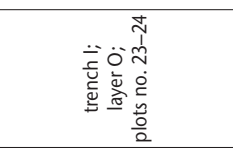 & 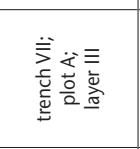 & 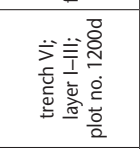 \\
\hline 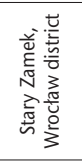 & 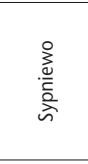 & 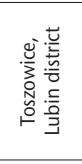 & 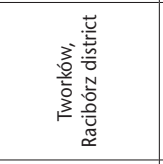 & $\frac{5}{\frac{5}{3}}$ & $\frac{.5}{\overline{0}}$ & 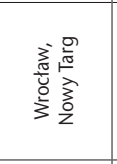 & 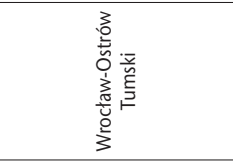 & 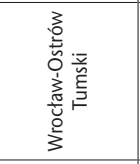 & 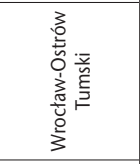 \\
\hline$\sim$. & - & $\sum_{\Sigma}^{j} \stackrel{m}{m}$ & $\frac{\bar{u}}{a}$ & -. & r. & 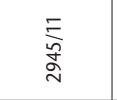 & $\frac{\hat{N}}{\hat{\sigma}}$ & $\frac{\circ}{\stackrel{\infty}{\infty}}$ & 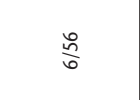 \\
\hline 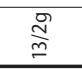 & \pm & $\stackrel{0}{5}$ & 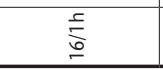 & 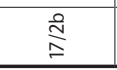 & 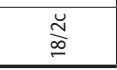 & $\stackrel{\widetilde{I}}{\check{I}}$ & $\stackrel{\text { N }}{\stackrel{N}{2}}$ & $\stackrel{u}{\stackrel{n}{N}}$ & 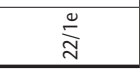 \\
\hline
\end{tabular}


One green ornamented bead from Wrocław and another one from Wolin, similar in terms of shape, size and decoration show remarkable similarities to those finds (fig. $1: a$; $2: b ; t a b .1:$ no. 17, 20). It is also worth noting that their estimated chronology is comparable (see Chronology of large beads).

The category of dark polychrome beads, decorated with glass threads in different shades can be discussed separately. Particular specimens differ from one another in shape or quality of production but the obvious common feature is that their body was made of dark (black, or brown less frequently) opaque glass and ornamented with white, yellow or red thread in the form of wavy or wavy and straight lines. Considering the area of Poland, only one bead from Wolin (fig. 2: c; tab. 1: no. 18) can be included in the group, and similar examples sometimes occur at cemeteries in Bohemia and Moravia (e.g Mař́k 2009, 97, pl. 60; Staššíková-Štukovská - Ungerman 2009, 149; fig. 5: 4)33, however, they presumably originated from regions outside the Slavic lands (see Large glass beads - local or foreign product?).

The bead from Tworków is analogously decorated yet quite different when it comes to shape. It is biconical, however, it is higher $(27.3 \mathrm{~mm})$ and much more massive that the other ones (diameter $29.8 \mathrm{~mm}-t a b .1$ : no. 16). The item was made of dark blue-black opaque glass. It is decorated with a yellow thread forming interweaving stripes of wavy lines between two surrounding lines (fig. $1: h$ ). Regarding colour of ornamentation and stylistic features it most resembles the bead from Wolin (tab. 1: no. 18).

The common feature of all large beads found in the area of Poland is the diligence of production. Visually, only one bead from Ciepłe and one shapeless specimen from Janów Pomorski (fig. 2: $d, i$ ) differ from the standard. In the case of the first one the reason for the unattractive look is the poor state of preservation, as it is emphasized that the item was manufactured with due diligence (Wajda 2019, 221). In the case of the latter it is assumed that it became deformed as a result of overburning (Dekówna-Purowski 2012, 218) ${ }^{4}$.

\section{Context of discovery of large beads}

In Poland large glass beads are being found in the areas of strongholds (tab. $1:$ no. 4, 5, 9-11, 20-22), settlements (tab. 1: no. 7, 8, 15, 15) and cemeteries (tab. 1: no. 1-3, 12-14). Nevertheless, it is remarkable that the finds concentrate within important centres (Gdańsk, Wolin, Janów Pomorski, Czersk, Łęczyca, Wrocław, Opole) and developing early towns (Grodków). Basing on distribution of glass beads (fig. 3), it may be assumed that they occur in places connected with trade and exchange, and along communication routes, such as the Odra (Tworków near Racibórz, Opole, Wrocław, Czeladź Wielka, Toszowice), Barycz (Sądowel) or Vistula rivers (Ciepłe).

\footnotetext{
${ }^{3}$ We will not thoroughly discuss the finds from whole Central Europe, because a preliminary analysis revealed that the beads that occur in particular regions, e.g. in Poland, Bohemia and Moravia, cannot be described using the same scheme. They differ in both the time and context of discoveries. Their social and cultural interpretation is also different. The selected examples are only supposed to illustrate the diversity of typologies, functions and context of discovery of beads.

4 The only worse quality bead comes from Přerov in Central Moravia. Numerous light-coloured spots in the black body of the item were noted, which indicates inappropriate melting of the glass paste (Procházka 2018, 159).
} 


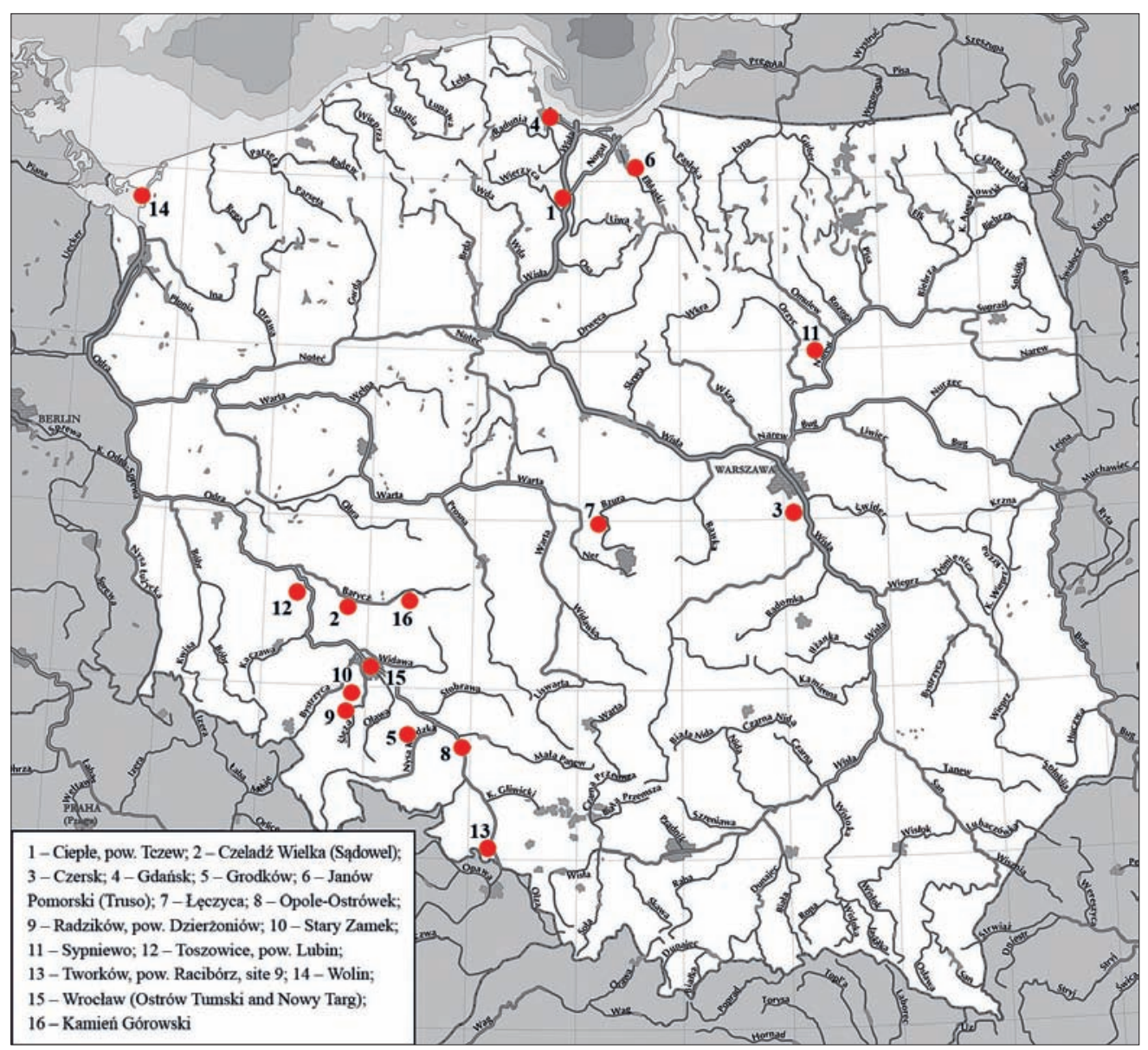

Fig. 3. Location of the study sites on a map of Poland.

It is also worth paying attention to the places where the beads were exactly discovered. Some of them were loose finds (tab. $1:$ no. 3, 6), some came from the topsoil layer (tab. 1 : no. 7) or from undefined context (tab. 1: no. 8, 15). It is remarkable that large part of the beads whose localization was precisely determine come from undeveloped area. All specimens from Ostrów Tumski in Wrocław were discovered outside buildings. A green bead decorated with coloured threads were found in an empty square (tab. 1: no. 20; Kaźmierczyk - Kramarek - Lasota 1979, 169-170). Unfortunately, precise context of discovery of the beads from trench VI and trench VII on Ostrów Tumski in Wrocław ${ }^{5}$ and the specimens from Wolin is unknown (tab. 1: no. 17, 18). In the area of a medieval street a greenish

\footnotetext{
${ }^{5}$ However, we assume that both of them were discovered outside buildings. Bead no. 6/56 was found within the area of plot dz. 1200d that encompasses the street and the remains of buildings. Specimen no. 281/60 was found, in turn, close to an impressive building interpreted as a pagan temple (Moździoch 2004,330-334), yet on plot A, which was only partly included in the building's area.
} 
bead from Gdańsk was found (Chmielowska 1960, 115). A massive specimen from Nowy Targ in Wrocław was discovered in an empty space associated with the existence of a market square in that place (Marcinkiewicz - Piekalski 2018, 163-177, fig. 92).

The specimens from Opole-Ostrówek occurred inside buildings. A bi-conical turquoise bead was discovered in house no. 18/I which was characterized by steady construction of both walls and floor. Its surface equaled $17.5 \mathrm{~m}^{2}$, which allows for including it in the group of larger buildings uncovered in that settlement level. Inside it, apart from the bead, other glass and bronze decorations, an artefact interpreted as a bronze needle case lid, a type II/3 spur and a limestone cone were found (Bukowska-Gedigowa-Gediga 1986, 210). The concentration of luxury goods (elements of armour, metal decorations, gemstones and glass) in the building's immediate vicinity, especially feature no. 69B and no. 19B, is also remarkable (Siemianowska 2017, 156). Less impressive finds come from house no. 59, in which a green spherical glass bead was discovered. Nevertheless, it was not a poor household. From the interior come women's accessories - several glass and carnelian beads and a tin temple ring as well as a lead barrel-shaped weight (Bukowska-Gedigowa-Gediga 1986,172 ). As a matter of fact, both beads were found beyond the area where the most representative finds at this site occurred, however, in this area quite a large concentration of horse-riding gear and trade-related artefacts was recorded (see Wachowski 1984, fig. 52; Bukowska-Gedigowa - Gediga 1986, fig. 64, 80).

The finds from cemeteries form a separate group. However, it should be emphasized that in the area of Poland such finds are relatively rare (see $t a b .1$ ), whole beads discovered in the settlement context dominate ${ }^{6}$. We will get back to the problem of presence of such objects in graves when discussing their function.

\section{Chronology of large beads}

Glass beads, often referred to as spindle whorls, are known in Europe since at least Roman period. At that time the most frequent were the beads with visible traces of spiral winding of glass, sometimes decorated with also spirally wound coloured thread. In the beginnings of the Early Middle Ages, large glass beads sometimes occur at cemeteries from the Migration period and the Early Middle Ages in Western Europe. Flattened spherical specimens (spindle whorl shape), made of green and yellow glass, decorated with a fine ornament composed of a multiplied combed glass threads, dominate there (Haevernick 1972; Tempelmann-Maczyńska 1985, 62-63, Taf. 13-14; Siegmann 2007, 280, Abb. 11; 2009, 151, Abb. 15) ${ }^{7}$. Several analogous objects dated to the period between 450 and 600 AD are know from Scandinavia (Haevernick 1972, 141; Steppuhn 1998, 76-77, Taf. 9).

\footnotetext{
${ }^{6}$ It is not typical of Central Europe. For example, in the Czech lands, large beads occur much more often at cemeteries (e.g. Krumphanzlová 1965, 164, obr. 1; Sláma 1977, 38, 35, Abb. 9: 19; Mař́k 2009, 97, tab. 60; Staššíková-Štukovská - Ungerman 2009, 149, obr. 5: 4; Schejbalová 2011, 37, 102-105, obr. 64). In strongholds and settlements they are very rare. One bead was discovered in Přerov (Procházka 2018, 159, fig. 19: 3), a larger one is also known from Chotěbuz-Podobora (Kouřil - Gryc 2014, 137, obr. 8), however, it is too subtle for the category of large beads and should be probably regarded as a larger decoration bead.

${ }^{7}$ https://pl.pinterest.com/pin/32369691043536741/?lp=true; https://research.britishmuseum.org/research/ collection_online/collection_object_details.aspx?objectId=411749\&page=1\&partId=1\&peoA=96389-3-12\& people=96389; https://www.metmuseum.org/art/collection/search/239921
} 
The fact that the specimens from earlier periods (mostly from the Roman period) ${ }^{8}$ are very similar to the early medieval large beads found in the area of Poland is remarkable. This applies to greenish or turquoise beads of corrugated surface as well as to large and smaller beads decorated of multi-coloured glass threads in the form of wavy, or less frequently straight lines (cf. fig. 1: a, h; 2: b, c; Tempelmann-Maczyńska 1985, types 225, 261, 263, 267, 293-303, 308). A number of analogies to the latter can be also found in early medieval Scandinavia (e.g. Arbman 1940, Taf. 120: 11, 122: 13, 123: 2, 37; Callmer 1977, pl. 1-7: No: B0110, B0160, B0200, B0520, B0530, B0640-0700, B111T, B2260-2270; Steppuhn 1998, Taf. 7: 16, 30, 34-37, 42; 8: 4; 9: 31; Farbtaf. 96 and 97). The similarity causes problems in determining the provenance and chronology of beads, especially in the case of those of undefined context of discovery.

In the analysed assemblage from the area of Poland, the bead from Tworków may be the oldest specimen. It was discovered at a site where the settlement phase embraced both the Roman period and the Early Middle Ages (Dębski 2014a; 2014b; Romańska 2014a; $2014 b$ ). The bead itself was discovered close to the finds associated with the Przeworsk culture, far from the concentration of early medieval items (see Dębski 2014a, fig. 2: 4; $2014 b$, fig. 1). That is how the discussed artefact was dated (phase C1b of the Roman period; Romańska 2014b, 129, fig. 96: 3). Determining the chronology of the bead from Janów Pomorski is also problematic, as it was discovered in the humus layer. It might be an early medieval object that should be dated to the ninth century, however, as M. Dekówna and T. Purowski $(2012,81)$ suggested, the specimen is similar in terms of composition of glass to the glass used in the Hallstatt, La Tène and Roman periods (see also Large glass beads - local or foreign product?).

Among the specimens of earlier chronology also one large bead from Wolin, discovered in a layer dated to 950-1000 (tab. 1: no. 17), can be mentioned. A similar one from Wrocław comes from a layer whose chronology was defined as the first quarter of the eleventh century (tab. 1: no. 20) $)^{9}$. To the eleventh - first quarter of the twelfth century (together with the whole cemetery) the specimen from Stary Zamek (tab. 1: no. 13) is dated. Similar chronology (fourth quarter of the eleventh and first half of the twelfth century) have the beads from the cemetery at Ciepłe (tab. 1: no. 1). The time when yellow and green spherical beads from Wrocław and Opole were deposited in the ground was probably from the fourth quarter of the eleventh century to the first half of the thirteenth century $(t a b .1$ : no. 11, 22), and for the ones made of turquoise glass it was the end of the eleventh century and generally the Early Middle Ages (tab. 1: no. 10, 21 ${ }^{10}$ ). Other greenish and turquoise 'corrugated' beads from the area of Poland are dated from the twelfth-thirteenth century to the first half of the fourteenth century (tab. 1: no. 4, 5, 14). Such a wide chronology indicates that those items were used also in the Late Middle Ages, which seems to be con-

\footnotetext{
8 The remark does not apply to the beads with combed ornament from the Migration period and the beginning of the Middle Ages. They have their own unique style that has no analogies neither in the Roman period nor in the younger phases of the Middle Ages.

9 A little younger chronology of the layer cannot be excluded, but even if the correction is taken into account, the item could not have been deposited later than in the mid-eleventh century.

${ }^{10}$ In the case of the specimen from Wrocław (tab. 1: no. 21) younger chronology cannot be excluded as well. The artefact was discovered in a layer in which spurs typical of the Late Middle Ages occurred, too (see Ostrowska 1963, fig. 1: 2, 3).
} 
firmed by recent discoveries. From a layer dated to the fourteenth century comes a lightcoloured checked bead from the excavations at Nowy Targ in Wrocław (tab. 1: no. 19).

Defining the chronology of other beads causes more problems. Undecorated light-green specimen from Toszowice is dated generally to the Early Middle Ages (tab. 1: no. 14). Precise chronology of the similar beads from Łęczyca and Czeladź Wielka (tab. 1: no. 3, 9) has not been determined, either. Relatively simple form of those non-decorated items does not allow us to refer to analogies, helpful in defining the time of their production/deposition. The remark applies also to other beads from Poland, as those are often unique, of inimitable style (tab. 1: no. 6, 9, 11, 12, 16-22). Dated broadly to the Middle Ages is the bead from Grodków or its immediate vicinity. Although it does not have direct analogies among other items of that kind as well, the ornament can be helpful in determining its chronology. The bead is decorated with multi-coloured circular lines and the motif of diagonal crosses between arms of which there are dots in various colours. Analogous motifs can be seen from the thirteenth century on knife handles (e.g. Kaźmierczyk 1970, fig. 27) and that is why we assume that the bead should be dated to the Late Middle Ages. However, it is also possible that this is an even younger specimen - from the Renaissance period, which can be indicated by the ornament composed of multi-coloured dots, very popular as a decoration painted on glass in the sixteenth and the beginning of the seventeenth century (e.g. Hess Husband 1997, 97, 99, 103, 135, 196, 200; Sedláčková - Rohanová et al. 2016, fig. 2, 10, 12). To the group of younger specimens also belongs the black bead decorated with white and yellow thread from Wolin. It comes from a layer dated to ca. 1300-1400 (tab. 1: no. 18). The situation is complicated by the fact that within the same stratigraphic unit early medieval and modern-period materials occurred as well, and the bead itself resembles with its decoration the specimen from Tworków, which may come from the Roman period.

The popularity of multi-coloured beads does not come to an end with the decline of the Middle Ages. Glass and glazed beads occur during the Modern Era in different parts of Europe as well. ${ }^{11}$

Even though colourful beads are rather rare finds, their chronology in the area of Poland is quite broad. The oldest examples can come from the prehistoric period. At sites located on or close to trade routes several specimens could have been present as early as in the ninth century, however, they became commonly used in the late tenth or eleventh century. It seems that this kind of artefacts spread in the area of Poland in direct connection with the so-called early-state breakthrough. They became most frequent in the period between the late eleventh and the thirteenth century, however, they were still used in the Late Middle Ages and the Modern era.

\section{Similar items made of different materials}

In the period when large glass beads appear in Poland also a number of objects of similar form can be found (fig. 4). In the case of similar clay products, when they are discovered at settlement sites, there is practically no possibility to distinguish them from spindle whorls

11 Glazed specimens: https://research.britishmuseum.org/research/collection_online/collection_object_details. aspx?objectId=33821\&partId=1\&images=true\&object=20241\&view=list\&page=6 
(e.g. Sláma 1977, 193, Abb. 32: 19, 33: 4). Nevertheless, similar items made of clay are sometimes found at cemeteries and described as beads, not spindle whorls. The cases when such objects were used as temple ring pendants may suggest their decorative function as well (e.g. Sláma 1977, 67, Abb. 19: 8).

Similarity to glass beads (especially specimens with corrugated, spirally wound surface) can be observed in the case of identical in terms of shape and decoration knobs made of amber (fig. 4: $l$ ). A form of similar shape (corrugated surface) is also often found among stone (fig. 4: $a-c, i-k)$, more rarely clay (fig. 4: $e-h$ ) and sometimes lead (fig. 4: d) spindle whorls (e.g. Hotubowicz 1956, fig. 82c; Bukowska-Gedigowa-Gediga 1986, fig. 15: 10, 66: 39, 40; 69: 41, 42; 85: 14, 88: 1, 90: 15, 116: 12, 19, 37; 125: 25, 27; Kaźmierczyk 1990, fig. 78: 2, 79: 79-14, 7-10, 17, 19, 22, 24; Marek - Kostelníková 1998, 189-190; Jagodziński 2010, 98-107, fig. 279, 286; Biermann - Kieseler-Nowakowski 2011, fig. 7e; Březinová - Přichystalová 2014, 184, pl. 5). M. Dekówna $(1970,36)$ mentions analogous items made of different materials (bone, stone) across the Mediterranean region.

The similarity provokes reflection on whether stone spindle whorls and amber knobs (?) were imitations of glass beads, or just the opposite. The analogies between glass and amber decorations were already noted in the case of signet rings that were characterized by identical form and very similar colour (cf. Pankiewicz - Siemianowska - Sadowski 2017, 43). The comparison of amber and glass beads also seems to suggest that the analogous form is not a coincidence, even though they differ in colour. The interpretation of their function is identical - they are regarded as decorations or spindle whorls (see Functions of large glass beads, also: Lisowska et al. 2017; Ciombor - Romanowicz 2019, 333-334). Both materials - glass and amber - were appreciated in the Early Middle Ages. In order to try to answer the question which material was used to produce beads earlier, it would be necessary to analyse their chronology. As it was already pointed out, large glass beads occur in the area of Poland mostly in the younger phases of the Early Middle Ages. Production of amber decorations (including large beads) yet began in the Baltic zone much earlier (Jagodziński 2010, 149-153, figs. 209-217; Ciombor - Romanowicz 2019, 346). On the other hand, due to different colour range and the fact that most glass beads were considered as imports (see also: below fragment), it is not possible to regard them as imitations of amber ones.

Large glass beads, especially those characterized by spiral winding of glass, resemble to a large extent clay and stone spindle whorls decorated with grooves running around. This type of decoration is commonly found within main stronghold centres of the Piast state where glass beads also occur (tab. 1). 'Corrugated' spindle whorls appear in Central Europe as early as in the ninth/first half of the tenth century (e.g. Marek - Kostelníková 1998, 189-190; Jagodziński 2010, 98-107, fig. 279, 286; Biermann - Kieseler - Nowakowski 2011, fig. 7e; Březinová - Přichystalová 2014, 184, tab. 5; Rębkowski 2019, pls. XLVIII: 19, LXI: 9, CIX: 25, CLXXIV: 2, 3; for dating see: Michczyński-Rębkowski 2019), or even in the early Slavic period (Parczewski 1989, pls. XVIII: 4, XXXVIII: 2, XLIII: 13; Poleski 2013, fig. 11: 4). Such early chronology and widespread occurrence exclude the possibility that they could be imitations of glass beads. The similarity of objects with grooved surface probably does not result from the desire to copy particular patterns but from using the same technological solution - a turning lathe in the case of 'classic' spindle whorls and amber knobs (e.g. Marek - Kostelníková 1998, 190; Březinová - Přichystalová 2014, 184), and spiral winding of glass in the case of glass beads. 
a

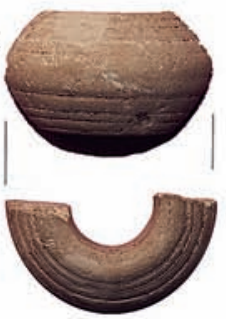

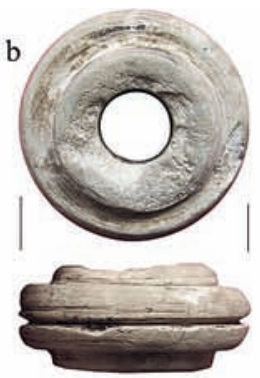

c

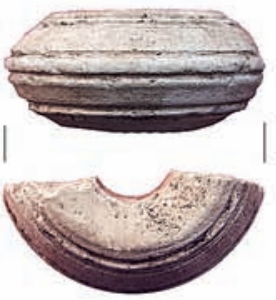

d
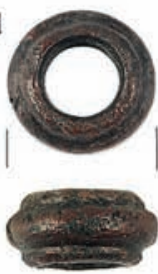

e

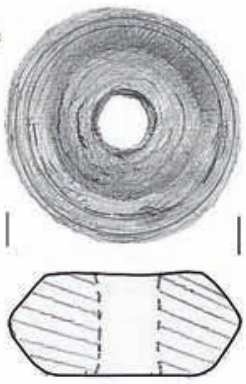

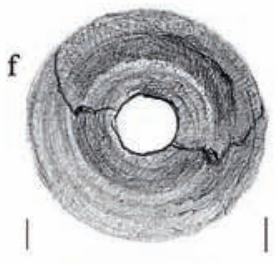

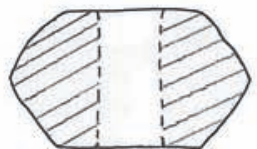

g
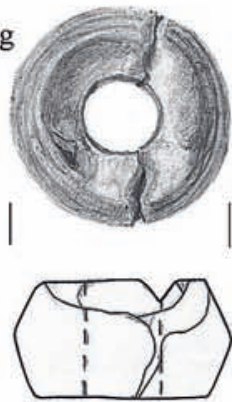
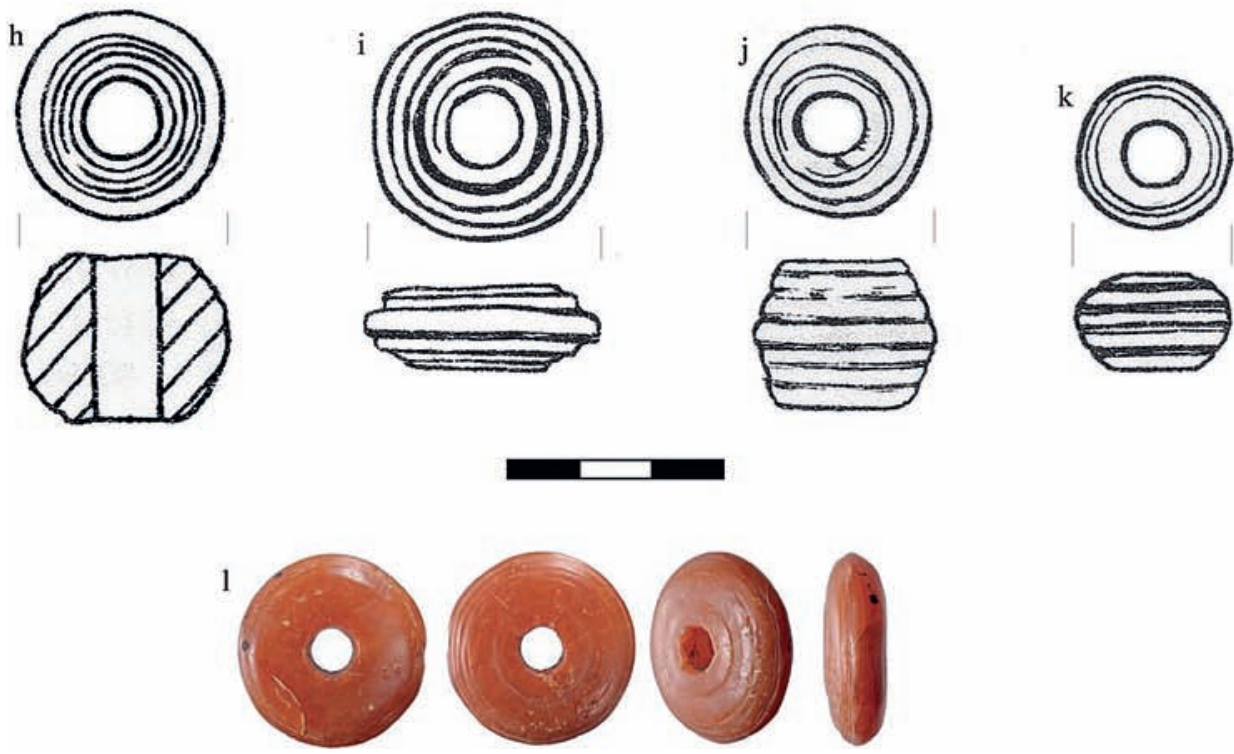

Fig. 4. Items (spindle whorls and beads) made of various materials: stone (a-c, i-k), lead (d), clay (e-h) and amber (I); a-g - Wrocław Ostrów Tumski; h-k - Opole Ostrówek; I - Janów Pomorski (Truso); $\mathrm{a}$ - trench III/86, building no. 7, layer $\mathrm{E} 3$; $\mathrm{b}$ - trench III/84, building no. 1 , layer $\mathrm{C} 1 ; \mathrm{c}$ - trench $\mathrm{III} / 85$, plots no. 67-69, 72-74, layer D1; d - trench I, plot 18, layer G; e - trench IIIF, layer B5; f - trench IIIF, layer B4; $\mathrm{g}$ - trench IIIF, layer B9; $\mathrm{h}$ - All layer, are 375; i - All layer, are 312; - All layer, are 343; k - Al layer, are 493. a-c - photos by E. Lisowska, d - photo by J. Sawicki; e-g - after Lisowska 2015, fig. 2; h-k - after BukowskaGedigowa - Gediga 1986, fig. 76, 125; I - after Jagodziński 2010, fig. 209: 4. 
In our opinion, analogies between the style of glass and amber beads as well as stone and clay spindle whorls result from general canons of decoration used for items of similar shape, size and probably also function (see Functions of large glass beads).

\section{Large glass beads - local or foreign product?}

In order to determine the provenance of large beads, physico-chemical composition of glass paste used for the production of the available specimens from Silesia (five objects) was analysed. The analyses carried out during earlier studies (Siemianowska 2020), and information contained in the literature were also used (Dekówna 1970, 21-25; DekównaPurowski 2012, tab. 5, 6; Wajda 2019, 255). The analyses revealed considerable differentiation of chemical composition of those objects (see tab. 2 and fig. 5). In the case of the decorated specimen from Ostów Tumski in Wrocław (fig. 1; tab. 1: no. 20), the body was produced of soda-lime-potassium glass (fig. 5: b), while the ornament was made of lime-potash-lead glass (yellow thread - fig. 5: c; tab. 1: no. 20) as well as lead-potash glass (brown and reddish thread - fig. 5: d; tab. 1: no. 20) ${ }^{12}$. The body of the specimen from Wrocław Nowy Targ was made of soda-lime natron glass with aluminum, potassium and chloride, and the decorative grid was made of soda lime natron glass with opacifying agent indicated by oxides of $\mathrm{PbO}$ and $\mathrm{SnO}(t a b .2)$. Different glass recipe in decorations was also recorded in the case of bead from Tworków (figs. $1: h ; 6$, and tab. 1: no. 16). The body was produced of soda-lime natron (?) glass coloured with iron (fig. 6: $a, c$ ), and the ornament was made of lead-potash glass, in which traces of calcium, magnesium, aluminum and iron compounds were noted (fig. 6: b; Siemianowska 2020, fig. 6). From soda-lime glass a black, yet absolutely different in terms of visual features, bead from Přerov (Procházka $2018,159)$ was also made.

Both greenish (turquoise) beads from Wrocław and Opole (fig. 1: b, c; tab. 1: no. 10, 21) were characterized by soda-lime natron glass recipe with high content of aluminum oxides and chloride (fig. 7, tab. 2) ${ }^{13}$, and in the case of the specimen from Opole also magnesium (fig. 7). Similar composition of glass (soda-lime natron) was noted in the case of the bead from Czersk (tab. 1: no. 4). The specimen from Gdańsk, in turn, was made of soda-lime-potassium (mixed alkali) glass (tab. 1: no. 5). It is worth noting that those four glass decorations are visually very similar and are some of the most common bi-conical turquoise beads found in the area of Poland. Moreover, the surface of three of them is shaped with spiral twists (cf. fig. 1: b; tab. 1: no. 4, 5, 10, 14; Dekówna 1970, 21-21, figs. 1-3). Among the beads with 'corrugated' surface, the most outstanding in terms of composition of glass is the specimen from Ciepłe, made of lead glass (tab. 1: no. 1).

\footnotetext{
12 We are aware of the method's limits. However, the painted stripes were so wide and well-preserved that it was possible to analyse particular colourful threads (fig. 5: a). In order to obtain reliable results the surface of the examined artefacts was delicately abraded, without damaging the paints yet removing the substances that could have gathered there when it was used or when it was deposited in the ground.

13 The increased chloride content is characteristic of blue and bluish glasses. That is why it is assumed that its presence influenced the colour of finished products as well (Pankiewicz - Siemianowska-Sadowski 2017, footnote 32).
} 


\begin{tabular}{|c|c|c|c|c|}
\hline Component & WOT $281 / 60$ & WOT $6 / 56$ & WNT 2945/11 (body) & WNT 2945/11 (ornament) \\
\hline $\mathrm{K}_{2} \mathrm{O}$ & 0.43 & 0.64 & 1.08 & 0.72 \\
\hline $\mathrm{CaO}$ & 7.71 & 0.47 & 6.85 & 3.58 \\
\hline $\mathrm{P}_{2} \mathrm{O}_{5}$ & 0.05 & 0.03 & 0.11 & 0.06 \\
\hline $\mathrm{SO}_{3}$ & 0.27 & * & 0.1 & 0.04 \\
\hline $\mathrm{Cl}_{2} \mathrm{O}$ & 1.49 & 0.06 & 0.95 & 0.63 \\
\hline $\mathrm{PbO}$ & * & 67.3 & 0.03 & 38.46 \\
\hline $\mathrm{Ag}_{2} \mathrm{O}$ & 0.01 & 0.02 & * & * \\
\hline $\mathrm{SnO}$ & 0.02 & 0.04 & 0.03 & 3.89 \\
\hline $\mathrm{Sb}_{2} \mathrm{O}_{3}$ & 0.02 & 0.06 & 0.2 & 0.07 \\
\hline $\mathrm{BaO}$ & 0.02 & 0.12 & 0.05 & 0.06 \\
\hline $\mathrm{Cr}_{2} \mathrm{O}_{3}$ & * & $*$ & * & 0.01 \\
\hline $\mathrm{Na}_{2} \mathrm{O}$ & 17.6 & 0.01 & 15.05 & 8.27 \\
\hline $\mathrm{SiO}_{2}$ & 68.3 & 30.11 & 72.08 & 40.65 \\
\hline $\mathrm{Al}_{2} \mathrm{O}_{3}$ & 2.74 & 0.51 & 2.55 & 1.83 \\
\hline $\mathrm{MgO}$ & 0.65 & 0.08 & 0.84 & 0.48 \\
\hline $\mathrm{As}_{2} \mathrm{O}_{3}$ & * & * & * & 0.01 \\
\hline $\mathrm{SrO}$ & 0.02 & 0.17 & 0.12 & 0.09 \\
\hline $\mathrm{Fe}_{2} \mathrm{O}_{3}$ & 0.6 & 0.13 & 0.3 & 0.6 \\
\hline $\mathrm{MnO}$ & 0.67 & 0.01 & 0.32 & 0.54 \\
\hline $\mathrm{CoO}$ & 0.03 & 0.02 & 0.01 & * \\
\hline $\mathrm{NiO}$ & 0.02 & * & * & 0.13 \\
\hline $\mathrm{CuO}$ & 0.07 & 0.05 & 0.11 & * \\
\hline $\mathrm{ZnO}$ & * & 0.26 & * & * \\
\hline $\mathrm{TiO}_{2}$ & 0.07 & 0.09 & 0.08 & 0.05 \\
\hline Total & 100.79 & 100.18 & 100.86 & 100.17 \\
\hline
\end{tabular}

Tab. 2. The results of chemical composition analyses of early medieval large glass beads from Ostrów Tumski (WOT) and Nowy Targ (WNT) in Wrocław. * - component not found.

From non-alkaline lead glass two spherical beads, green and yellow, were made. They resemble 'regular' beads that commonly occur at Silesian strongholds (tab. 1: no. 10, 21). The same recipe was used for the production of small yellow and green beads as well as rings and band-rings in identical colour (cf. fig. 1 : $d$; tab. 1: no. 22; tab. 2; PankiewiczSiemianowska - Sadowski 2017, 33-34, cat. no. A2-5, 9, 10, 12-14; B1, 3, 22, 27, 29, 30-34, 36, 38, 42-46, 54-56, 58, 59, 60, 63-71, 92-95, 98-101). Chemical composition is another common feature of large beads and 'ordinary' decorations.

Recipes of lead content glass, both alkaline and non-alkaline, and lead-potassium glass are widely considered to be characteristic for Central European production in the younger phases of the Early Middle Ages (Olczak 1968, 181-183; Dekówna 1980, 283-300; Dekówna - Purowski 2012, 163-165; Černá - Tomková 2017, 202-204; Pankiewicz Siemianowska - Sadowski 2017, 33-35; Siemianowska - Pankiewicz - Sadowski 2019). Taking into account the above criterion, only the find from Ciepłe and the recently mentioned simple non-decorated beads can be regarded as local products. Due to the fact that lead glass was spread across vast areas of Central, Western and Eastern Europe as well as the Middle East and Northern Africa (Olczak 1968, 181-183; Dekówna 1980, 283-300; Wedepohl - Krueger - Hartman 1995, Tab. 1; Robertshaw et al. 2010; Dekówna - 

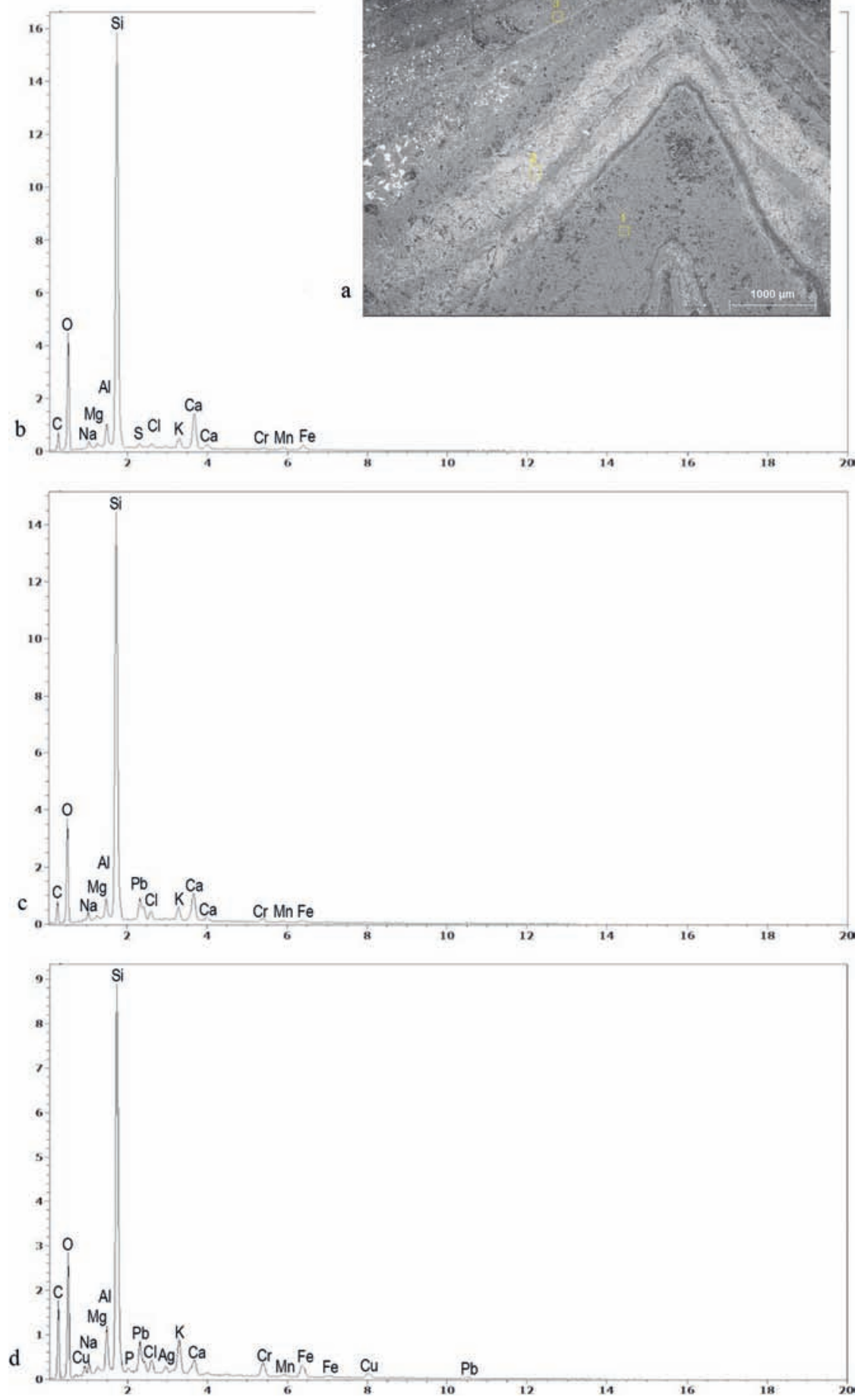

Fig. 5. A microscopic photo of a large bead from Wrocław Ostrów Tumski (inv. no. 63b/77) with a marked place of the performed analyses (a) and the results of EDS analyses (b-d): b-body; $c$-yellow thread; $\mathrm{d}$-brown (red-brown) thread. 

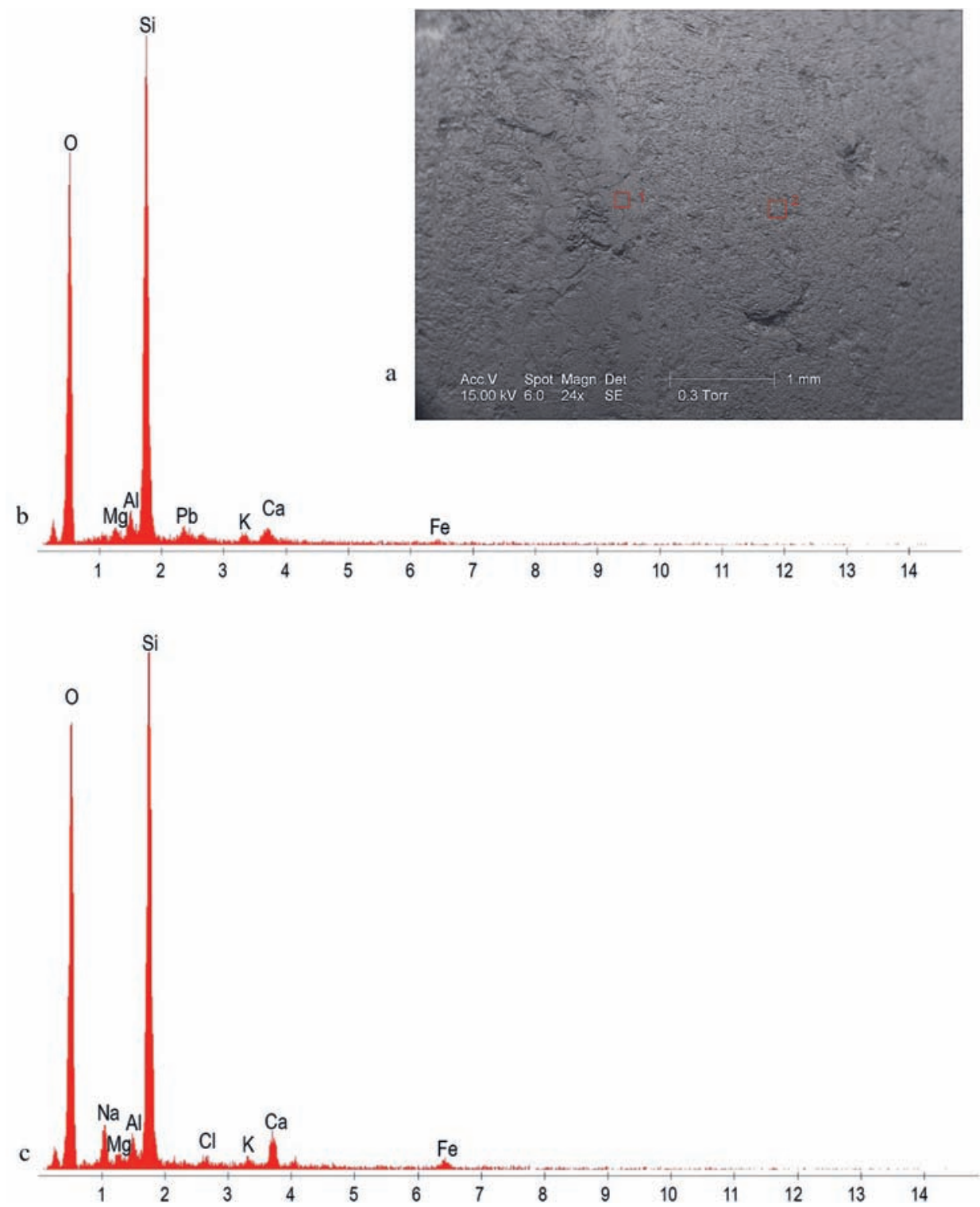

Fig. 6. Results of EDS analyses of a large bead from Tworków (inv. no. 9/c/1): a - microscopic photo with the marked place of the analyses; $b$ - analysis of the ornament; c - analysis of the body.

Purowski 2012, 163-165; Mecking 2013, 646-659, fig. 3, 5; Krueger 2014; Pollak 2017; Pankiewicz - Siemianowska - Sadowski 2017, 33-35), and the specific form of the beads their foreign origin must be taken into consideration.

Soda-lime natron glass, soda-potassium-lime and lime-potassium-soda glasses are regarded as imports from the Mediterranean region, Asia Minor and Central Asia (Dekówna 1970, 25-26; 1980, 220; Dekówna - Purowski 2012, 95; Wajda 2013, 96; Černá - Tom- 


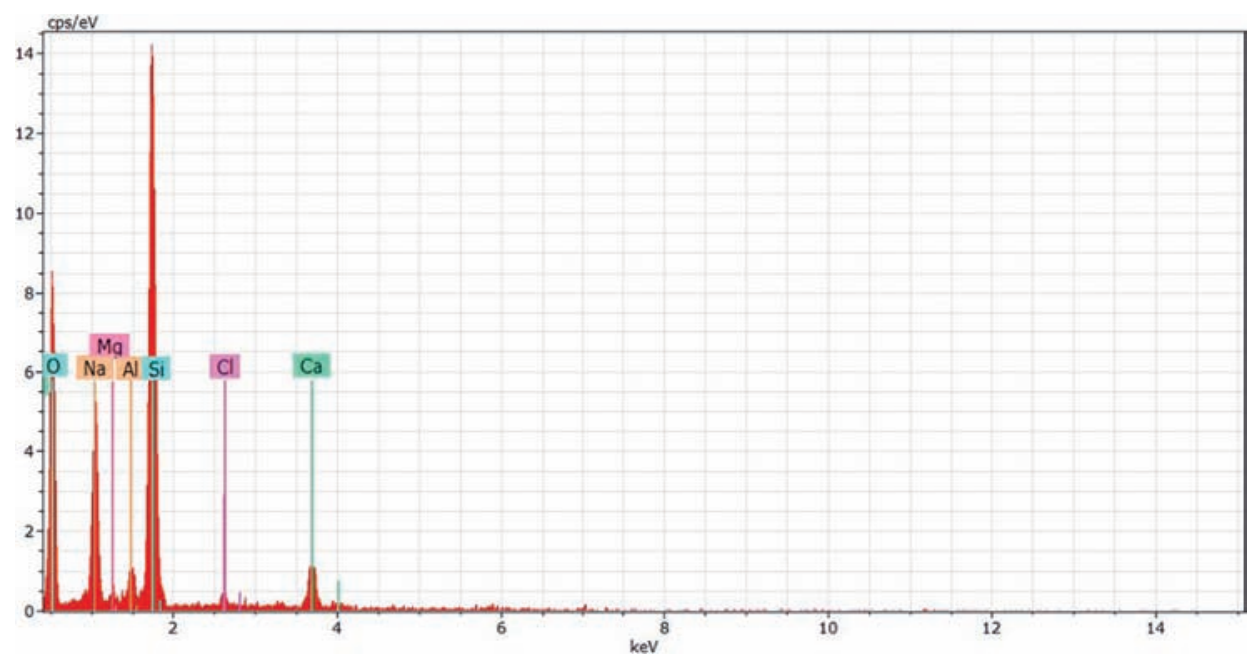

Fig. 7. Results of EDS analyses of a large bead from Opole Ostrówek (inv. no. 356/58).

ková 2017, 203, 206; Pankiewicz - Siemianowska - Sadowski 2017, 35-35). This type of glass recipes were also known in Western Europe, but due to the relatively late chronology of Polish finds their western origin is highly improbable, as since the tenth century potassium-lime-silicate glasses begin to prevail in the West (Dekówna 1970, 25-26; DekównaPurowski 2012, 153-154). Since the thirteenth century potassium glass becomes common in local production (Černá 2016; Černá - Tomková 2017, 205; Siemianowska 2020; Siemianowska - Sadowski 2019), which makes local origin of the youngest specimens (Czersk, Wrocław Nowy Targ) made of soda-lime natron glass questionable. South-eastern provenance of bi-conical beads decorated with spiral grooves that are found in the area of Poland is indicated also by their typological similarity to the specimens from the area of present-day Syria, Georgia and Armenia (Dekówna 1970, 26-37, fig. 4 and 5).

Typological similarity is the second criterion that should be considered when trying to determine the potential place of production of beads. It had been already mentioned that similar items occurred in Europe in the earlier periods and analogous patterns were used, e.g. in early medieval Scandinavia (see Chronology of large beads). Because of evident cultural relationships between the Polish lands and the Scandinavian region, it seems quite natural to seek the origins of some products there. In the case of beads of 'corrugated' surface it has no justification. Letting alone the used resources, no formal analogies were recorded. Such specimens do occur in Scandinavia ca. 600 AD (Haevernick 1972, 141), but in the Viking Age both large beads and 'ordinary' small beads in similar style are very rare (see Arbman 1940, Taf. 120-124; Callmer 1977, pl. 2-22; Steppuhn 1998, Taf. 1-10, Farbtaf. $)^{14}$. Therefore we keep our opinion that in the area of Poland such specimens should be interpreted as imports from the Middle East.

\footnotetext{
14 In the assemblages of glass decorations published in these works only one bead characterized by spiral winding of the glass paste is similar (see also: Steppuhn 1998, Farbtaf. 25). Nevertheless, 'spindle whorls' of this kind are recorded at Scandinavian sites from the Viking Age as well (see also https://pl.pinterest.com/pin/1493929126144 $27528 / ?$ lp=true).
} 
The second group of beads that can have originated from Scandinavia are those green or black ones decorated with colourful threads (for analogies, see Chronology of large beads). The bead from Tworków is not taken into account due to its uncertain chronology. Other dark-coloured bead ornamented with white and green threads (fig. 2: c), discovered in Wolin, has close analogies among glass decorations from Scandinavia, including beads and large beads type B0670-B0700, regarded as objects produced in that region (see also Callmer 1977, 35-104, pl. 6) ${ }^{15}$. However, the chronology of the specimen from Wolin is problematic, as it is not certain whether it is an early or late medieval artefact. We do not know its chemical composition either (tab. 1: no. 18).

Scandinavian analogies can also be found for the second bead from Wolin and the similar specimen from Ostrów Tumski in Wrocław (fig. 1: $a ; 2: b$ ). Scandinavian finds of medium- and large-sized beads of similar stylistic features can be mentioned, however, no direct analogies were found (e.g. Arbman 1940, Taf. 11: 1c, 11: 11n; 122: 13a, c; Callmer 1977, types B640-724, 35-36, 51, pl. 14). It is also remarkable that among green beads from Scandinavia there were no specimen that could be regarded as large beads and most of them were decorated not with wavy lines yet with applied loops (Callmer 1977, 35-36, 51, Colourplate III). It is assumed that the specimens in such colours, decorated with wavy lines (group Bd) could have been local (Scandinavian) products (Callmer 1977, 83-84, 96). Chemical composition of the bead from Wolin is unknown yet, the one from Wrocław was made of lime-potassium-soda glass. The recipe is not typical of Scandinavia and was considered to have been of eastern origin. Making a clear interpretation is complicated due to the fact that in Scandinavia in the ninth-tenth century various types of glass was used, including different kinds of soda-based recipes, whose presence could have resulted from importing or re-melting glass as well as different origin of finished goods (Dekówna 1980, 172-198; Kronz et al. 2015). In this context the early chronology of the bead from Wrocław (first quarter of the eleventh century) could confirm its northern origin.

On the other hand, analogies to large and medium-sized glass beads decorated with colourful threads occurred in the early medieval Middle East (Liu 2012). Typologically, they are also similar to those from the Roman period, which can be caused by the continuity of patterns introduced in the Middle Eastern glass-working workshops.

In order to answer the question of the origin of early medieval beads from the basin of the Vistula and Odra rivers it is worth to look again at the circumstances in which the objects appeared in the area of Poland and their distribution. The similarity between the beads from Wolin and Scandinavian finds may not be a coincidence. Despite the lack of direct analogies at the northern and western coast of the Baltic Sea for one of the beads, northern origin of the beads from that centre, considering intensive contacts between Wolin and Scandinavia (Stanistawski 2013, passim), seems to be very likely.

The bead from Wrocław that is similar to one of those discovered in Wolin, is dated to the first decades of the eleventh century. This period (from the late tenth to the mid-eleventh century) is the time of the highest frequency of items representing Scandinavian style on Ostrów Tumski in Wrocław (Jaworski et al. 2013, passim). On the other hand, this is the moment when the first wave of glass objects identified on the basis of chemical composition and stylistic features as imports, presumably from the Middle East, appeared

15 In this work: size criteria p. 35, macroscopic description p. 37-51, provenance p. 96-104. 
at this site. It is worth emphasizing that those are often exclusive and unique finds in the area of Poland (Pankiewicz - Siemianowska - Sadowski 2017, 63-64). That is why we tend to consider the glass bead as an item imported from this cultural zone, not Scandinavia.

Most of the glass beads from the area of Poland can be regarded as imports, to a large extent from the East or South-East. The objects, usually finely made and often decorated, were undoubtedly valuable in the early medieval reality. According to tenth-century Arabic sources, one green glass bead bought by Ruthenian traders was worth one dirham (Lewicki 1953, 118). Massive decorative glass beads must have been worth much more.

The time of the highest frequency of the occurrence of glass beads in the area Poland late eleventh to thirteenth century - is not a coincidence either. Its chronology coincides with the second wave of eastern imports reaching the area of the basins of the Vistula and the Odra. At that time, those are mostly imports from Ruthenia. They manifest themselves with the presence of elements of arms and horse-riding gear of Ruthenian type, spindle whorls made of Ovruch schist, metal decorations and miniature axes and also glass products (beads for mosaics, glass vessels, bracelets, game pieces etc.; Jaworski 1990, 58-59; Wachowski 2001, 170, fig. 18a, c-e; Wołoszyn 2004; Lisowska 2013, 136-138; PankiewiczSiemianowska - Sadowski 2014, 199-203; 2017, 65-67; Siemianowska 2020; in print). ${ }^{16}$ As objects imported from this cultural zone sometimes glazed eggs and rattles (yet not without doubts) are regarded (Ślusarski 2004, 80-83, 103-105, 108; Siemianowska 2008, 69-73; Siemianowska - Pankiewicz - Sadowski in print). Together with items directly from Ruthenia, the Polish lands were reached by objects from places located much further to the South-East, which is evidenced by discoveries of coins and seals from the Middle East at that time (Wołoszyn 2004, 246-250). The presence of most glass beads in this part of Europe is probably connected with this wave of influences.

\section{Functions of large glass beads}

\section{Large glass bead as an element of a necklace}

The possibility of using large beads as elements of necklaces is somehow determined by the material they were made of and their decorative character. Sometimes, despite considerable size and width of the hole, which could indicate other functions, a bead was automatically classified as a decoration, basing on the material (e.g. Grygiel-Stasiak - Trojan 2014, 196-197, fig. 118: 23).

In some cases context of discovery clearly indicates that glass beads served such function. The occurrence of large beads as elements of necklaces was recorded already at cemeteries from the Migration period and the beginning of the Early Middle Ages in the regions

\footnotetext{
${ }^{16}$ It must be emphasized that some of the mentioned items may come from the eleventh century. To the eleventhtwelfth century miniature axes are generally dated (Wołoszyn 2004, 252). Also, the chronology of spindle whorls made of Ovruch slate, 'Ruthenian' glass beads and glazed eggs-rattles cannot be limited to the period before the twelfth century, however, it is visible that the frequency of such finds (e.g. beads, bracelets, Ovruch slate spindle whorls inceases in the twelfth century (see Lisowska 2013, catalogue; Pankiewicz - Siemianowska - Sadowski 2017, 65-67), while other types (e.g. glass vessels) only start to appear (Pankiewicz - Siemianowska - Sadowski $2014,199-203)$. The first wave of Ruthenian imports connected with the early Piast state is not taken into consideration either.
} 
located west of the Elbe (e.g. Siegmann 2007, 280, Abb. 11). They were used in a similar way in the area of Poland during the discussed period. Bi-conical, spirally wound specimens, discovered together with other beads in grave no. 70 in Stary Zamek (WachowskiDomański 1992, 27, 69, fig. 43) and grave no. 34 in Ciepłe (Ratajczyk 2013, fig. 7; Wajda 2019,225 ), are good examples. Similar specimen, yet made of amber instead of glass ${ }^{17}$, was discovered in grave no. 62 in Kałdus (Markiewicz 2008, 149-154, pl. 14). Several monochrome beads of 17-19 $\mathrm{mm}$ in diameter were found in necklaces from the cemeteries in Radzików (grave no. 3 - Wachowski 1975, 121-122, fig. 20) and Ciepłe (grave no. 41 Wajda 2019, 221).

The examples prove that massive beads, made of either glass or amber, served as a dominant in the whole necklace, being its most impressive element. Reconstructions of different necklaces made of glass beads or other materials clearly indicate that the largest and therefore most representative specimens (one or several examples) took central position in the whole string of beads. Smaller 'ordinary' beads were placed at sides and at the back of a necklace (e.g Wachowski 1975, fig. 20; Dekówna 2007, no. 372, 396, 399-403; Markiewicz 2008, figs. 4, 5, 7, 9, 18, 26, 29, 31, 36, 44, 48, 49, 52, 53, 63, 65, 73, 94, 95, pls 1a, 2a, 3b, 4b, 5, 7, 8b, 9b, 10a, 13, 14, 16; Ratajczyk 2013, fig. 7).

It is also worth noting that massive beads as elements of necklaces can be found in the area of Poland in very rich sets of jewelry. The necklace from Kałdus, whose central element was a large bead, was composed of over 800 glass beads of different kinds. Less representative were the necklaces from Radzików ( 54 beads), Stary Zamek ( 25 beads) and Ciepłe (19 and 8 beads), however, they were also made of different beads, mostly decorated.

Presence of such a necklace that must have been a very costly thing in a grave can be regarded as an indicator of high social status of those who owned it. It may be also confirmed by other items discovered in graves where strings of beads with a central massive bead were found. In grave no. 3 in Radzików three rings, including one with a gemstone, and a bracelet made of bronze were discovered (Wachowski 1975, 121-122). Grave no. 62 from the cemetery in Kałdus with the remains of a child aged 4-6, apart from a necklace also contained a glazed ceramic rattle and a trapezoid pendant, a temple ring and seven bronze belt fittings (Markiewicz 2008, 154). A less impressive necklace from grave no. 34 in Ciepłe was accompanied by four temple rings and other decorations (Ratajczyk 2013, fig. 7). The artefacts do not seem very impressive in comparison to grave goods discovered in the discussed cemetery, being one of the richest in the area of Poland. Nevertheless, it must be emphasized that large beads together with smaller beads come from the younger - definitely less rich - phase of the use of the cemetery and considering this particular period they can be regarded as objects of special meaning.

\section{Large glass bead as a spindle whorl}

Large glass beads were often interpreted as spindle whorls, or at least such an option was taken into consideration (Krumphanzlová 1965, 164; Dekówna 1970, 38-43; Haevernick

\footnotetext{
17 The whole necklace is described as made of glass, however, in the description of particular types of beads there is no mention on large beads. Nevertheless, there is information on a specimen made of amber present in the grave.
} 
1972, 136-138; Pankiewicz-Siemianowska-Sadowski 2017, 44; Procházka 2018, 159). This is the result of macroscopic similarity of these items, often different only in terms of materials used for their production. Direct analogies refer to the form of the object itself, its size and the presence of a large hole - cylindrical or slightly widening, funnel-shaped in the central part. Most of early medieval spindle whorls are bi-conical with ends flattened at the hole, or sometimes spherical and flattened, referred to as lenticular or barrel-shaped (e.g. Kaźmierczyk 1990, 193; Dominiczak-Głowacka 2008, 250-255; Březinová - Přichystalová 2014, 177-180), however, also more complex typology of those object is proposed (e.g. Marek - Kostelníková 1998, 174-176, 181-185, Abb. 2, 6).

Apart from similar form, large glass beads bear much metric resemblance to spindle whorls made of other materials. Most of such beads have a diameter of $18-35 \mathrm{~mm}(25-26 \mathrm{~mm}$ on average) and are 9.6-27.3 $\mathrm{mm}$ high (16-17 $\mathrm{mm}$ on average, see $t a b .1)$. In this respect they very much resemble spindle whorls whose diameter usually varies between 22 and $33 \mathrm{~mm}$, and the height between 12 and $22 \mathrm{~mm}$ (Kamińska - Nahlik 1958, 35; Kaźmierczyk 1990, List of stone spindle whorls IZestawienie przęślików kamiennych/; Dominiczak-Gtowacka 2008, List of well-preserved spindle whorls IZestawienie prześlików zachowanych w całości/; Březinová - Př́chystalová 2014, 184; Lisowska 2015, tab. 1; Janowski 2019, 300-301, fig. 123). The size differences among spindle whorls result from both specified territorial preferences and the differentiated specific weight of the substances that were used for production (clay, stone, lead, bone etc.). It is associated with the need of proper balancing, however, the weight of particular objects is also not the same. In the case of stone spindle whorls from Wrocław it equaled 5-19 g (Kaźmierczyk 1990, 186). In Gdańsk the standard weight of spindle whorls was $7-15 \mathrm{~g}$ for lighter ones and 20-23 $\mathrm{g}$ for heavier ones (Kamińska - Nahlik 1958, 35). The weight of spindle whorls from Wolin ranged from $6.4 \mathrm{~g}$ to $78.7 \mathrm{~g}$, however, most of them contained within the range of 10-30 g (Janowski 2019, 302, fig. 125-131). In Mikulčice spindle whorls whose weight was 5-16 g prevailed (Marek - Kostelníková 1998, Abb. 9). In Břeclav-Pohansko the weight was considerably varied, yet the average equaled $14.4 \mathrm{~g}$ (Březinová Prichystalová 2014, 181-182). It is assumed that the differences in the weight of elements used for weighting a spindle was tightly connected with the thickness of weave of woolen thread that was supposed to be achieved (e.g. Kamińska - Nahlik 1958, 35; Dekówna 1970, 39; Janowski 2019, 303-307). Spindle whorls of different sizes, made of various material have one common feature - the size of the hole which fits the spindle. In the case of spindle whorls from Poland of all types it was 7-12 mm, in overwhelming majority $8-10 \mathrm{~mm}$. Only in several cases it was narrower than $7 \mathrm{~mm}$, yet assuming that the second hole of the funnel-shaped channel was wider (Dekówna 1970, footnote 46; Kaźmierczyk 1990, List of ... ; Foltyn 1998, 92-93; Dominiczak-Gtowacka 2008, List of ... ; Lisowska 2015, tab. 3; Janowski 2019, 303, fig. 125). Smaller size was recorded in the case of the assemblages of spindle whorls from Mikulčice and Břeclav-Pohansko, where specimens whose hole diameter equaled 7-9 mm dominated. Spindle whorls with the hole diameter of $5 \mathrm{~mm}$ and smaller occurred sporadically (Marek - Kostelníková 1998, 195; Březinová - Př́chystalová 2014, 180).

The figures are different considering beads. In the case of ca. one third of them the diameter of the hole oscillates around $5 \mathrm{~mm}$, or sometimes it is even less (tab. 1: no. 2, 4, 5, 8, 10, 17, 20, 21). It seems to be too little to interpret the items as spindle whorls. Even though M. Dekówna (1970, 39-40) claims that this might be connected with the Mediterranean origin of glass beads where thinner fabrics were worn, it is not very likely that such preferences could have been adopted in the climatic conditions of Central Europe.

Nevertheless, it is possible to see the correlation between massive glass beads and 'ordinary' spindle whorls, especially in the area of Poland where there is a possibility of more precise dating. Not including the specimens of uncertain chronology, deposition of most of such artefacts was dated to the younger phases of the Early Middle Ages and to the Late Middle Ages. In the case of the discussed region it is the time of considerable differentiation of spindle whorls. Before the tenth century this category of objects was not 
very much varied. Those made of clay, sometimes decorated dominate (e.g. Foltyn 1998, 92-93, tab. 9; Jaworski 2005, 262), while stone ones are rare. Only at few sites that were characterized by unique material culture a wider spectrum of materials from which this kind of objects were made have been recorded. The conclusion applies to the places connected with trade and exchange, such as seaside centers functioning within the so-called Baltic economic zone (e.g. Wolin, Truso, see Jagodziński 2010, fig. 279, 283-286; Janowski 2019, 293-298, fig. 114-122) and several strongholds in the interior (e.g. Jaworski 2005, 262, 272-284, fig. 142). It does not seem to be a coincidence that at coastal locations the oldest large glass beads were recorded (cf. tab. 1: 7, 17). On the other hand, the assortment of spindle whorls from 'early Polish' sites, such as the strongholds in Wrocław or Opole, looks completely different. Within those centres, functioning since the second half, or even more likely since the end of tenth century at the earliest, clay spindle whorls still prevail, however, stone ones made of different kinds of stones became common as well. Moreover, spindle whorls made of lead, bone, clay mixed with graphite as well as clay ones covered with coloured glaze or even wax ones occur, too (Hotubowicz 1956, 194-195, fig. 82; Jaworski 1990, 75-75; pl. XI: o-s; Kaźmierczyk 1990, 182-193; Dominiczak-Głowacka 2008, 247-248; Lisowska 2013, 134-142). Among them, specimens made of foreign materials appear, e.g. there is a large group of spindle whorls made of Ovruch schist, imported from Ruthenia. In the situation when in the area of the early Piast state luxury decorations appeared and glass became more commonly used in jewelry production, it seems natural that it could have been used as the material to produce another, symbolic object - a spindle whorl (cf. Wotoszyn 2004, 251; Březinová - Přichystalová 2014, 190).

The context of occurrence is fundamental for the theory on glass beads being used as spindle whorls. As it was already mentioned, a significant part of the discussed category of finds from Poland was discovered outside densely built-up areas (see Large glass beads as other decorations, knobs/amulets). The distribution of spindle whorls finds looks completely different, as they were mostly found in houses (Kaźmierczyk 1990, 182; Moździoch 2001, figs. 8, 12, 17, 24, 26, 28, 33, 35, 39, 41, 43; Lisowska 2015, tab. 1). At this point, it is worth taking a look again at the specimens that had been discovered in features. As it was revealed, both massive beads from Opole were accompanied by pieces of women's jewelry, indicating quite high social status of the owners of such precious items. In feature 18/I, apart from the hypothetical spindle whorl, woolen threads and felt were found. Even more traces of spinning come from the neighboring features 19 and 20, in which four fragments of spindles, three limestone spindle whorls as well as numerous finished and semi-finished goods (fabrics, threads, strings and raw wool) were discovered (BukowskaGedigowa-Gediga 1986, 210-211). Together with a green spherical massive bead, five spindles, a spindle whorls and a woolen thread were found in house no. 59. Accessories related to wool-processing were recorded in all buildings in the area (no. 18C - two spindle whorls, no. 66 - four spindles, four spindle whorls; no. 67 - two spindles, a spindle whorl; no. 68 - a spindle whorl, woolen string; Bukowska-Gedigowa-Gediga 1986, 168, 172,174 , 176, figs. 68-69). Such a set of spinning tools, however, does not prejudge the function of the glass bead. This specimen has a funnel-shaped, rather narrow channel whose diameter equals 3-5 $\mathrm{mm}$. The state of preservation of the item (a crumbled half) does not allow us to determine whether the hole in the bead had an outlet, or the channel narrowed without any outlet $(f i g .1 d)$. Originally the object was reconstructed without a through hole, which makes the possibility of using it as a spindle whorl highly doubtful 
(Bukowska-Gedigowa - Gediga 1986, fig. 68: 17). This issue and other possible ways of using this item will be discussed later.

In the discussion concerning the question whether massive glass beads were spindle whorls or decorations one more possibility should be also taken into consideration - that they could have served both functions. It may be indicated by the examples of placing pink slate spindle whorls in necklaces (Dekówna 2007, no. 396).

\section{Large glass beads as other decorations, knobs/amulets}

Discussing the function of massive glass beads as elements of jewelry, the attention was also focused on the fact that those could have been not necessarily massive beads serving as a kind of a dominant in a necklace, yet decorative knobs (Dekówna 1970, 38-41). This kind of knobs could have been attached to clothes, e.g. to a belt (Siegmann 2009, 151, Abb. 15) or being used as a hanging head decoration. Such pieces of jewelry can be seen, for instance, on mosaics from Ravenna (e.g. Siegmann 2009, 151). However, it is not certain whether those pendants were made of glass, gemstones or other materials. Examples of attaching single spindle whorls (?) to massive temple rings, so that they became an integral part of the decoration, are also known (e.g. Sláma 1977, Abb. 19: 8).

The function as a glass knob can be, paradoxically, indicated by the context of discovery of some large beads, because, as it was stressed, they were often found far from houses, unlike 'classic' spindle whorls and 'ordinary' glass beads. In the case of pieces of glass jewelry (beads and rings) found in the streets and squares it was assumed that they could have been traded, however, it seems that local products made of lead glass were rather sold (Pankiewicz - Siemianowska - Sadowski 2017, 42-43; Siemianowska - Pankiewicz-Sadowski 2019). Massive beads were probably items of foreign origin, high value and unique style, which makes it rather unlikely that they should be associated with trade. Therefore, they should be perceived as lost items (fully-preserved specimens: tab. 1: no. 5 , 16-18, 21) or objects that had been damaged and abandoned (tab. 1: no. 7, 19, 21, 22). Losing one piece suggests that those could have been decorative knobs indeed.

It is also taken into consideration that a single bead could have served as a decorative ending of a braided hairstyle. This way of wearing it (in this case made of amber, not glass) is reconstructed on the basis of a grave find from Uniradze in Pomerania (Kostrzewski 1962, 170, fig. 89). Nevertheless, there was no information why it was interpreted as a hairstyle element. As M. Dekówna (1970, footnote 56) pointed out, their holes were too small to be suitable for pinning hair.

Small holes in some of the glass knobs make the possibility of using them as spindle whorls at least questionable, especially that this was characteristic of a considerable number of glass beads (see Large glass bead as a spindle whorl). Smaller hole did not cause any problems if a bead was supposed to be used as a knob. On the contrary, such a decoration looked more elegant and worked better as a pendant.

The occurrence of single glass decorations in graves is also remarkable. A larger bead of $14 \mathrm{~mm}$ in diameter was discovered in grave no. 6/6a in Tomice in Lower Silesia. It was a double grave which contained the remains of a young woman and a new-born child. The bead was found at the child's bones (Romanow - Wachowski-Miszkiewicz 1973, 174, 204, fig. 94b). It is worth noting that even if other massive beads discovered at cemeteries in the area of Poland were parts of necklaces, they occurred in children's graves (infans I- 
Kałdus; infans II - Stary Zamek; Wachowski-Domański 1992, 69; Markiewicz 2008, 154) ${ }^{18}$. Also other finds from Central Europe may confirm using large beads as knobs/amulets. A glass bead of over $2 \mathrm{~cm}$ in diameter, made of dark glass and decorated with a yellow wavy line, was discovered in grave no. 185 in Kanín near Libice, under a jaw of a woman at the age of maturus (Mař́k 2009, 97, pl. 60). The specimen had a wide hole, but due to its deformity it could not have been used as a spindle whorl. It was probably a knob-like decoration, worn on the head. This suggests that massive beads, apart from being valuable items, could have served symbolic and magical functions.

Some massive beads are also known from male graves from the Roman period, Migration period and the beginning of Early Middle Ages. They sometimes occur with swords and other elements of arms (so-called sword beads) and in this context they are treated as amulets (Tempelmann-Maczyńska 1985, 62-63). Their presence in female graves in the younger period (ninth-eleventh century) indicates that their function changed at that time, however, it does not exclude magical role of large beads in graves.

\section{Large glass beads as game pieces}

The function of massive glass beads as game pieces has been considered years ago (Dekówna 1970, footnote 51).

It was already stressed that glass beads are relatively often discovered outside buildings. They are sometimes found in the streets or on empty yards, which could suggest, apart from accidental losing (see Context of discovery of large beads), using them as game pieces. Concentrations of this kind of objects (wooden balls, chess pieces, etc.) are often noted in empty areas where people spent their free time (Pankiewicz. 2019, 151, 155, figs. 2, 3; Siemianowska 2017; in print).

Macroscopic similarity between some massive beads and game pieces is also worth noting. This applies first of all to spherical, single-coloured specimens (fig. $1: d, e$ ). They resemble board game pieces made of different materials - bone, antler, teeth, ivory, wood, terracotta, amber and other gemstones and also glass (Persson 1976; McLees 1990, 43-48; Jagodziński 2010, 149, 153, figs. 210, 221, 282; Breyer-Jonquay 2012, 19; Kimball 2013, Fig. 1-2; Gustavsson - Hennius - Ljungkvist 2015, 51; Siemianowska in print). The size of glass beads and pear-shaped game pieces is also similar. The presence of a hole in glass beads does not necessarily exclude the possibility of using them in games, as some game pieces had a hole that was used to attach them to the board (Jagodziński 2010, 154, fig. 221). In the case of spherical glass specimens this was a narrow, funnel-shaped hole, with or without an outlet - it is not clear (tab. 1: no. 11, 22). The presence of such a recess is especially typical of pear-shaped game pieces, among which there are specimens with a through hole, which makes them similar to spindle whorls (see also McLees 1990, 43-48, 188-192). Another features that hnefatafl pawns and massive beads have in common is the chronology of those finds in Wrocław and Opole and almost identical chemical composition (cf. tab. 1: no. 11, 22 and Siemianowska in print). The context of finds, material and shape of the discussed category of artefacts similar to 'regular' game pieces used for board games such as hnefatafl, originating from the tradition of the Roman world and

\footnotetext{
18 The gender and age of the individual from Radzików, whose grave contained a necklace with a large central bead. Basing on the grave goods, it can be assumed that it was a female (Wachowski 1975, 121-122).
} 
Germanic tribes as well as for board games referred to as ludus latrunculorum (Adamczyk 2012, 65; Kimbal 2013, 62-63; Siemianowska in print), does not prejudge such function. Although glass flattened-spherical, semi-spherical and spherical pawns (token-like) with through holes do sometimes occur, their chronology is much earlier and they have a flat basis. ${ }^{19}$ The most similar in morphological aspect could be pawns discovered in graves no. 523 and no. 750 in Birka at the Swedish coast, dated to the ninth century (Arbman 1940, Taf. 147, 148; Berglund 1970) or tenth-century specimens from grave no. 3 in Valsgärde (Graham-Campbell 1980, 24, 212) ${ }^{20}$. The similarity to game pieces can also be seen in discoidal examples, especially those decorated with surrounding ribbing, which makes them similar to flattened 'corrugated' massive glass beads (see Dekówna 1970, figs. 1, 2b, 4a, b, 5 and Khamaiko 2018, fig. 7). Discoidal pawns were sometimes fitted with a wider hole and that is why it was assumed that they could have been re-used as spindle whorls (McLees 1990, 62, 204-207). In the light of traces identified on glass beads the specimens similar to spindle whorls could have also served as game pieces (see Function of large glass beads in the light of microscopic analysis).

\section{Function of large glass beads in the light of microscopic analysis}

Four beads from Wrocław - Ostrów Tumski, Wrocław - Nowy Targ, Opole-Ostrówek and Toszowice were subjected to a microscopic analysis (fig. 8; tab. 1: no. 11, 15, 19, 20).

The surface of bead from Toszowice is strongly destroyed, covered in pittings and a layer of oxidized glass (figs. $1: g ; 8: e, f$ ). Due to the poor state of preservation, no traces of use were observed. After taking a closer look at the item, it was noted that the edges at the wider side of the hole are slightly rounded and blunt (fig. 8: e), while at the narrower side they are slightly sharper (fig. $8: f$ ). It can be an indication that the object was used as a spindle whorl. In fact, it would be a rather subtle weight for a spindle but it fits into the size range of spindle whorls. On the bead from Nowy Targ in Wrocław traces of polishing were clearly visible. They were mainly visible at one side of the hole, and they were so intensive that a thick coat of glass forming the ornament has worn off during the use (fig. 8: $c, d$ ), and on the surface near the channel a small recess appeared (fig. 8: c). It is worth noting that on the other side of the hole the polished spots were almost invisible. Micro-traces recorded on this specimen leave no doubts and suggest that the item functioned as a spindle whorl. Its metric features seem to confirm that. The bead from Nowy Targ was the most massive in the whole collection. Its overall diameter equaled $35 \mathrm{~mm}$, the diameter of the channel $(11 \mathrm{~mm})$ and the height $(14 \mathrm{~mm})$, which means it could compare to the largest spindle whorls (see Large glass bead as a spindle whorl).

In terms of size the green decorated bead from Ostrów Tumski in Wrocław could be included in the group of spindle whorls (tab. 1: no. 20). At this item polished spots were recorded right next to the hole, on both sides of the channel (fig. 8: $g$ ). Subtle traces of polishing were also noted on the surface of the bead, precisely, on the polar flattening at the hole (fig. 8: h). Microscopic analysis proved that this specimen was used as a spindle

\footnotetext{
${ }^{19}$ See also http://www.jocari.be/proddetail.php?prod=je32b_pion-0200-F1934-10.88; Skomsvoll 2012, 82-89.

${ }^{20} \mathrm{http} / / /$ hem.bredband.net/b512479/Hnefatafl_by_Sten_Helmfrid.pdf
} 
a

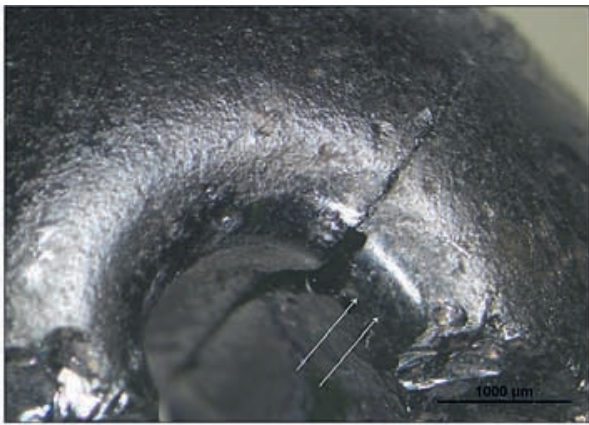

c

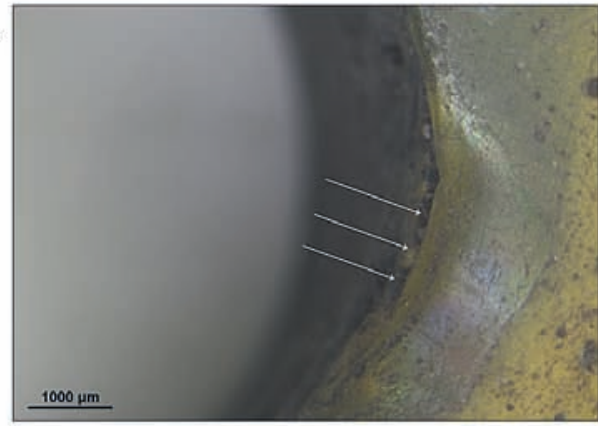

e

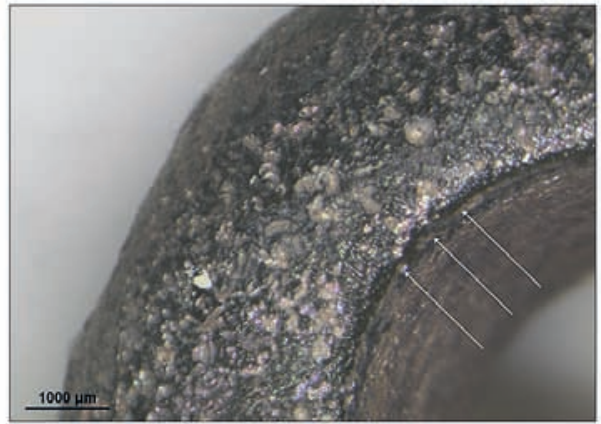

g

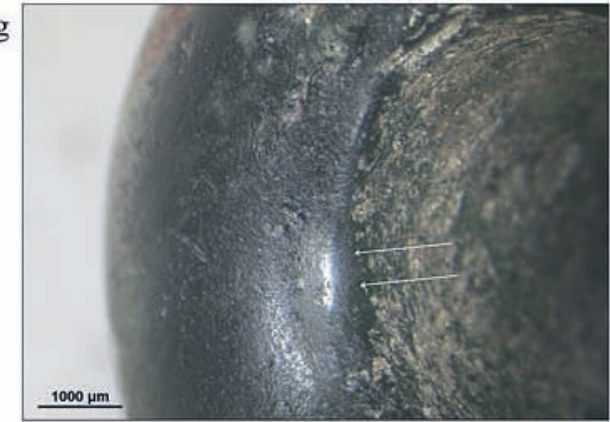

b

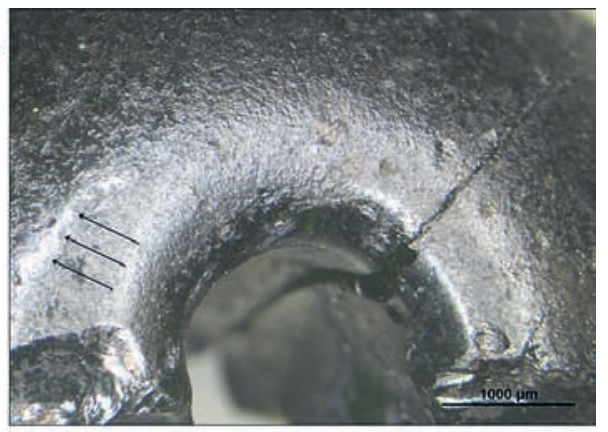

d

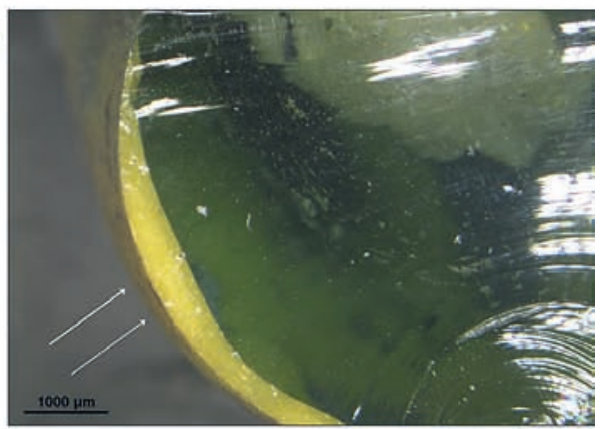

f

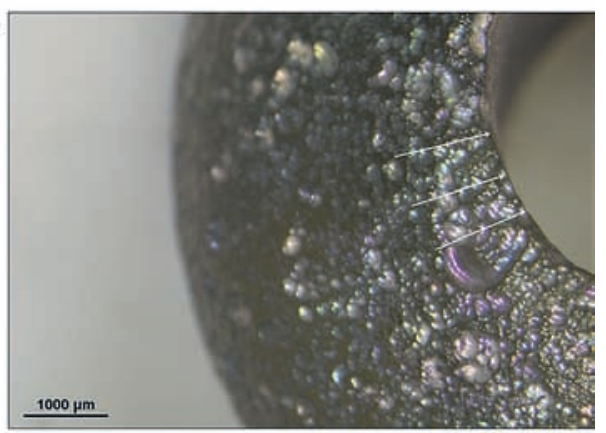

h

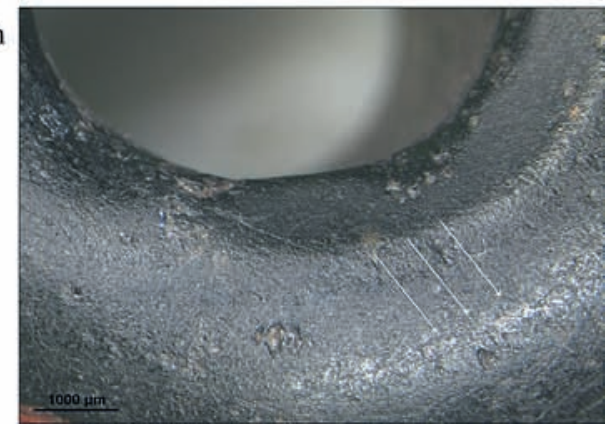

Fig. 8. Microscopic images of large glass beads: a-b - Opole Ostrówek, layer C2, are 311, m 8j, building no. 59 ; $c-d-$ Wrocław Nowy Targ, layer 19, are 54, quarter C; e-f-Toszowice, Lubin district, context of find unknown; g-h - Wrocław Ostrów Tumski, trench I, layer O, plots no. 23-24. Photos by A. Pankiewicz. 
whorl, however, there is a possibility that it was also used for other purposes, e.g. as a game piece. Analogous traces are visible on the last of the analysed beads, i.e. the spherical bead from Opole-Ostrówek. At the wider hole outlet clear yet uneven polished spots could be seen (fig. 4: a). Slightly polished areas were also visible at some distance from the hole, on the polar flattening of the object ( fig. 8: $b$ ). The context of discovery of this bead seemed to confirm its function as a spindle whorl, however, the shape of the item, different from traditional early medieval spindle whorls, and first of all, too small diameter of the hole raised doubts (see above). Considering one more important characteristic - a long crack that appeared already in the Middle Ages - one more hypothesis can be forwarded. We assume that the item functioned as a spindle whorl yet not as it was originally supposed to be used - worn as a decorative knob or as a pawn, and that is the reason why it got broken. The examples of using spindle whorls and game pieces interchangeably were discussed by other scholars (e.g. McLees 1990, 42).

Subtle traces of polishing at both ends of the last two described massive beads indicate that they also could have functioned as pawns. Microscopic observations allow for classifying glass beads as spindle whorls, however, they also suggest other ways of use.

\section{Conclusion}

Basing on the analysis of the context of finds, metric data and traces of use it was stated that massive glass beads did not serve one particular function, but they could have been used as spindle whorls, a single piece of jewelry, an element of a necklace or even a game piece. Regardless of their specific role, those were special items that accentuated the social prestige of its owner. Both their form and chemical composition of the material that was used for the production suggest that those were mostly imports, most probably from the Middle East and the Mediterranean region, and in some cases maybe from Scandinavia. Most of the analysed beads are very neatly made and decorated on the surface. Their value is dictated also by the weight of glass, which was a valuable material.

For the interpretation of function of glass beads the moment they appeared in the area of Poland is of key importance. Letting alone the specimens of uncertain chronology that can come from prehistoric periods, most beads can be dated from the late tenth century to the thirteenth century, however, there are several examples from later periods. The time when they appeared in the basin of the Vistula and the Odra is usually associated with social and cultural changes during the period when the Piast state was forming. At that time the need for various kinds of luxury goods increases, including typically female accessories - jewelry and spindle whorls. The presence of massive beads in the twelfth-thirteenth century is on the one hand a standard in material culture - yet a standard for the elites, and on the other hand they can be perceived as the evidence of vivid trade contacts with Ruthenia, through which also different products from the regions located further south-east reached Polish lands.

Nevertheless, there is no evidence that glass beads could have been a model for imitations made of other materials - e.g. stone spindle whorls with crimped decorations. It rather seems that the style is an expression of general tendencies in that period as well as similar functions and forms of those objects. Without a shadow of a doubt, massive beads were special items that were supposed to provide material and symbolic value. 
The publication realised within the framework of the project 'The development of central centers of the Piast state in the $10^{\text {th }}-12^{\text {th }}$ centuries on the example of the Wroctaw Ostrów Tumski' financed by Narodowe Centrum Nauki/the National Science Centre granted under the contract UMO-2017/27/B/HS3/00554.

The analyses of composition of glass were financed from research projects financed by the National Science Centre: Early Polish glass-making in the light of finds from Silesian stronghold complexes, agreement no. UMO-2013/10/E/HS3/00121, and Social and economic changes of the incorporation period in the light of glass finds from Silesian residential centres, agreement no. UMO-2013/09/N/HS3/01001.

We would like to thank for help Agata Witkowska and Krzysztof Demidziuk from the Archaeological Museum in Wroctaw.

\section{References}

Adamczyk, P. 2012: Gry skandynawskie. In: A. Stempin ed., Magia gry - sztuka rywalizacji, Poznań: Muzeum Archeologiczne w Poznaniu, 65-72.

Arbman, H. 1940: Birka I. Die Gräber. Tafeln. Uppsala: Almqvist \& Wiksel.

Berglund, L. 1970: 'Hnefatafl - en gåta?'. Saga och sed: Kungl Gustav Adolfs akademiens årsbok 1970, 80-92.

Biermann, F. - Kieseler, A. - Nowakowski, D. 2011: Od ogniska do zniszczenia pożarem. Grodzisko w Klenicy, gm. Bojadła, w świetle wyników nowych badań wykopaliskowych. In: A. Jaszewska - A. Michalak eds., Ogień - żywioł ujarzmiony i nieujarzmiony, Zielona Góra: Stowarzyszenie Naukowe Archeologów Polskich, Oddział Lubuski, 329-348.

Breyer, C. - Jonquay, S. 2012: Les jeux du monde Nordique La famille du Hnefatafl Semblables et différents. Histoire et Images Médiévales 28, 16-24.

Březinová, H. - Přichystalová, $R$. 2014: Úvahy o textilní výrobě na Pohansku na základě analýzy nálezů textilních fragmentů a předmětů souvisejících se spřádáním a tkaním. Památky archeologické 105, $155-214$.

Bukowska-Gedigowa, J. - Gediga, B. 1986: Wczesnośredniowieczny gród na Ostrówku w Opolu. Wrocław: Zakład Narodowy im. Ossolińskich.

Callmer, J. 1977: Trade Beads and Bead Trade in Scandinavia, ca. 800-1000 A.D. Bonn: Rudolf Habelt Verlag.

Chmielowska, A. 1960: Wyroby szklarskie z X-XIII wieku na stanowisku 1 w Gdańsku. In: J. Kamińska ed., Gdańsk wczesnośredniowieczny 3, Gdańsk: Gdańskie Towarzystwo Naukowe, 105-158.

Ciombor, K. - Romanowicz, P. 2019: Artefacts made of amber. In: M. Rębkowski ed., Wolin. The Old Town. Vol. II. Studies on Finds, Szczecin: Instytut Archeologii i Etnologii PAN, 323-347.

Černá, E. 2016: Stř̌edověkě sklárny v severozápadních Čechách. Mittelalterliche Glashütten in Nordwestböhmen. Most - Praha: Ústav archeologické památkové péče SZ Čech - Archeologický ústav AV ČR.

Černá, E. - Tomková, K. 2017: On the road from the Early to High Middle Ages: Glass of the 9th-13th centuries in Bohemia. Archaeologia Polona 55, 189-210.

Dębski, A. 2014a: Osadnictwo wczesnośredniowieczne. In: D. Minta-Tworzowska ed., Badania archeologiczne na terenie „Zbiornika przeciwpowodziowego Racibórz Dolny na rzece Odrze, województwo śląskie (polder)". Tom III/1. Tworków, stanowisko 9 (ZRD 9), Poznań - Wrocław: Instytut Archeologii i Etnologii PAN, etc., 233-282.

Dębski, A. 2014b: Osadnictwo wczesnośredniowieczne. In: P. Bobrowski ed., Badania archeologiczne na terenie „Zbiornika przeciwpowodziowego Racibórz Dolny na rzece Odrze, województwo śląskie (polder)". Tom III/2. Tworków, stanowisko 9 (ZRD 9), III sezon badań, rok 2014, Poznań - Wrocław: Instytut Archeologii i Etnologii PAN, etc., 81-107.

Dekówna, M. 1970: Uwagi o funkcji i pochodzeniu niektórych wczesnośredniowiecznych przedmiotów szklanych znalezionych na terenie Polski. Studia z dziejów rzemiosła i przemysłu 10, Wrocław: Zakład Narodowy im. Ossolińskich, 21-45.

Dekówna, M. 1980: Szkło w Europie wczesnośredniowiecznej. Wrocław: Zakład Narodowy im. Ossolińskich.

Dekówna, M. 2007: Wczesnośredniowieczne ozdoby szklane ze zbiorów Państwowego Muzeum Archeologicznego, na tle ogólnej problematyki rozwoju szklarstwa. In: W. Brzeziński ed., Skarby wieków średnich. Katalog wystawy, Warszawa: Państwowe Muzeum Archeologiczne, 52-87. 
Dekówna, M. - Purowski, T. 2012: Znaleziska związane ze szklarstwem oraz okazy z kwarcu ze stanowiska Janów Pomorski 1. In: M. Bogucki - B. Jurkiewicz eds., Janów Pomorski, stan. 1. Wyniki ratowniczych badań archeologicznych w latach 2007-2008. Tom I: 3. Analizy, Elbląg: Muzeum Narodowe w Szczecinie, 65-260.

Dominiczak-Głowacka, D. 2008: Przęśliki z wczesnośredniowiecznego grodziska w Grzybowie, w województwie wielkopolskim nieopodal Wrześni. Studia Lednickie 9, 243-273.

Foltyn, E. M. 1998: Podstawy gospodarcze wczesnośredniowiecznej społeczności plemiennej na Górnym Śląsku. Katowice: Wydawnictwo UŚ.

Graham-Campbell, J. 1980: Viking artefacts: a select catalogue. London: British Museum Publications.

Grygiel, R. - Stasiak, W. - Trojan, M. 2014: Gród łęczycki w świetle badań archeologicznych. In: R. Grygiel T. Jurek eds., Początki Łęczycy. Tom II. Archeologia o początkach Łęczycy, Łódź: Muzeum Archeologiczne i Etnograficzne w Łodzi, 11-587.

Gustavsson, R. - Hennius, A. - Ljungkvist, J. 2015: Are many Vendel and Viking Period gaming pieces made of whale bone?. Fornvännen 110, 51-54.

Haevernick, T. E. 1972: Nadelköpfe vom Typ Kempten. Germania 50, 136-148.

Hess, C. - Husband, T. 1997: European glass in the J. Paul Getty Museum. Los Angeles: The Museum.

Hołubowicz, W. 1956: Opole w wiekach X-XII. Katowice: Wydawnictwo Śląsk.

Jagodziński, M. 2010: Truso. Między Weonodlandem a Witlandem. Elbląg: Muzeum Archeologiczno-Historyczne

Janowski, A. 2019: Clay artefacts. In: M. Rębkowski ed., Wolin. The Old Town. Vol. II. Studies on Finds, Szczecin: Instytut Archeologii i Etnologii PAN, 291-322.

Jaworski, K. 1990: Wyroby z kości i poroża w kulturze wczesnośredniowiecznego Ostrowa Tumskiego we Wrocławiu. Wrocław: Volumen.

Jaworski, K. 2005: Grody w Sudetach (VIII-X w.). Wrocław: Instytut Archeologii Uniwersytetu Wrocławskiego.

Jaworski, K. - Lisowska, E. - Pankiewicz, A. - Stanisławski, B. 2013: Artefacts of Scandinavian origin form the Cathedra Island (Ostrów Tumski) in Wrocław. In: S. Moźdzoich et al. eds., Scandinavian culture in medieval Poland, Wrocław: Instytut Archeologii i Etnologii PAN, 279-314.

Kamińska, J. - Nahlik, A. 1958: Włókiennictwo gdańskie X-XIII w. Łódź-Wrocław: Zakład Narodowy imienia Ossolińskich.

Kaźmierczyk, J. 1970: Wrocław lewobrzeżny we wczesnym średniowieczu, cz. 2. Wrocław etc.: Zakład Narodowy im. Ossolińskich.

Kaźmierczyk, J. 1990: Kamień w kulturze Ostrowa Tumskiego we Wrocławiu w wiekach X-XIII. Wrocław Warszawa: Volumen.

Kaźmierczyk, J. - Kramarek, J. - Lasota, C. 1979: Badania na Ostrowie Tumskim we Wrocławiu w 1977 roku. Silesia Antiqua 21, 119-182.

Khamaiko, N. 2018: Gaming pieces from recent excavations of the Kyiv Podil. In: A. Stempin ed., The Cultural Role of Chess in Medieval and Modern Times. $50^{\text {th }}$ Anniversary Jubilee of the Sandomierz Chess Discovery. Poznań: Muzeum Archeologiczne w Poznaniu, 149-156.

Kimbal, J. J. L. 2013: From Dróttinn to King. The role of Hnefatafl as a descriptor of Late Iron Age Scandinavian culture. Lund Archaeological Review 19, 61-76.

Kokora, K. 2019: Glass artefacts. In: M. Rębkowski ed., Wolin. The Old Town. Vol. II. Studies on Finds, Szczecin: Instytut Archeologii i Etnologii PAN, 191-220.

Kostrzewski, J. 1962: Kultura prapolska. Poznań: Instytut Zachodni.

Košta, J. - Tomková, K. 2011: Olivovité korálky v raně středověkých Čechách a jejich postavení ve středoevropském kontextu, Památky archeologické 102, 307-354.

Kouřil, P. - Gryc, J. 2014: Hradiska 10.-12. století na severní Moravě a v českém Slezsku. In: K. Chrzan et al. eds., Funkcje grodów w państwach wczesnośredniowiecznej Europy Środkowej. Społeczeństwo, gospodarka, ideologia, Wrocław - Głogów: Instytut Archeologii i Etnologii PAN - Państwowa Wyższa Szkoła Zawodowa w Głogowie, 99-171.

Kronz, A. - Hilberg, V. - Simon, K. - Wedepohl, K. 2015: Glas aus Haithabu. Zeitschrift für Archäologie des Mittelalters 43, 39-58.

Krueger, I. 2014: Islamisches Bleiglas und mīnā. Journal of Glass Studies 56, 61-84.

Krumphanzlová, Z. 1965: Skleněné perly doby hradištní v Čechách, Památky archeologické 56, 161-188.

Lewicki, T. 1953: O cenach niektórych towarów na rynkach wschodniej Europy w IX-XI w. Kwartalnik Historii Kultury Materialnej 1, 112-132. 
Lisowska, E. 2013: Wydobycie i dystrybucja surowców kamiennych we wczesnym średniowieczu na Dolnym Śląsku. Wrocław: Instytut Archeologii Uniwersytetu Wrocławskiego.

Lisowska, E. 2015: Pozostałe wyroby ceramiczne z wykopu IIIF na Ostrowie Tumskim we Wrocławiu. In: A. Limisiewicz - A. Pankiewicz eds., Kształtowanie się grodu na wrocławskim Ostrowie Tumskim. Badania przy ul. św. Idziego. In Pago Silensi. Wrocławskie Studia Wczesnośredniowieczne 1, Wrocław: Instytut Archeologii Uniwersytetu Wrocławskiego, 285-292.

Lisowska, E. - Zamelska-Monczak, K. - Kolenda, J. - Gunia, P. - Łydżba-Kopczyńska, B. 2017: Multidisciplinary approach to the provenance investigations of early medieval amber finds discovered in Santok and Milicz. Fontes Archaeologici Posnanienses 53, 221-240.

Liu, R. 2012: Islamic glass beads. Ornament 36/1, 58-70.

Marcinkiewicz, K. - Piekalski, J. 2018: Przemiany placu w późnym średniowieczu i w czasach wczesnonowożytnych w świetle stratygrafii nawarstwień. In: J. Piekalski - K. Wachowski eds., Rytm rozwoju miasta na kulturowym pograniczu. Studium strefy placu Nowy Targ we Wrocławiu, Wrocław: Instytut Archeologii Uniwersytetu Wrocławskiego, 152-194.

Marek, O. - Kostelníková, M. 1998: Die Spinnwirtel aus Mikulčice. In: L. Poláček ed., Studien zum Burgwall von Mikulčice 3, Brno: Archeologický ústav AV ČR, 171-326.

Markiewicz, M. 2008: Biżuteria szklana z wczesnośredniowiecznych cmentarzysk strefy chełmińsko-dobrzyńskiej. Mons Sancti Laurentii 4. Toruń: Wydawnictwo Naukowe Uniwersytetu Mikołaja Kopernika.

Mař́k, J. 2009: Libická sídelní aglomerace a její zázemí v raném středověku. Dissertationes archaeologicae Brunenses / Pragensesque 7. Praha: Univerzita Karlova.

McLees, Ch. 1990: Games people played - Gaming-pieces, boards and dice from excavations in the medieval town of Trondheim, Norway. In: Fortiden i Trondheim bygrunn: Folkebibliotekstomten. Meddelelser $\mathrm{nr}$ 24, Trondheim: Riksantikvaren, Utgravningskontoret for Trondheim.

Mecking, O. 2013: Medieval lead glass in Central Europe. Archaeometry 55, 640-662.

Michczyński, A. - Rębkowski, M. 2019: Chronology of the cultural deposits. In: M. Rębkowski ed., Wolin. The Old Town. Vol. I. Settlement Structure, Stratigraphy \& Chronology, Szczecin: Instytut Archeologii i Etnologii PAN, 95-113.

Moździoch, S. 2001: Analiza przestrzenna rozmieszczenia tzw. zabytków wydzielonych w wykopie VI na Ostrowie Tumskim we Wrocławiu. Manuscript in the archiv of Ośrodek Badań nad Kulturą Późnego Antyku i Wczesnego Średniowiecza IAE PAN in Wrocław.

Moździoch, S. 2004: Wrocław-Ostrów Tumski in the Early Middle Ages. In: P. Urbańczyk ed., Polish Lands at the Turn of the First and the Second Millennia, Warszawa: Instytut Archeologii i Etnologii PAN, 319-338.

Olczak, J. 1968: Wytwórczość szklarska na terenie Polski we wczesnym średniowieczu. Studium archeologiczno-technologiczne. Wrocław: Zakład Narodowy im. Ossolińskich.

Ostrowska, E. 1963: Badania na wrocławskim grodzie w 1960 roku. Sprawozdania Archeologiczne 15, 268-271.

Pankiewicz, A. 2019: An attempt to identify places associated with trade and exchange in early medieval strongholds in the example of Ostrów Tumski in Wrocław. Přehled výzkumů 60-2, 145-160.

Pankiewicz, A. - Siemianowska, S. - Sadowski, K. 2014: Wczesnośredniowieczne naczynia szklane pochodzenia wschodniego z Wrocławia Ostrowa-Tumskiego i Opola-Ostrówka. Śląskie Sprawozdania Archeologiczne 56, 191-206.

Pankiewicz, A. - Siemianowska, S. - Sadowski, K. 2017: Wczesnośredniowieczna biżuteria szklana z piastowskich ośrodków grodowych Śląska (Wrocław, Opole, Niemcza). In pago Silensi. Wrocławskie Studia Wczesnośredniowieczne 3. Wrocław: Instytut Archeologii Uniwersytetu Wrocławskiego.

Parczewski, M. 1989: Żukowice pod Głogowem w zaraniu średniowiecza. Głogów - Wrocław: Dolnośląskie Towarzystwo Społeczno-Kulturalne.

Persson, J. 1976: Spel och dobbel. In: A. W. Mårtensson ed., Uppgrävt förflutet för PKbanken i Lund. Archeologica Lundensia. Investigationes de antiqvitatibus urbis Lundae VII, Lund: Kulturhistoriska museet, 379-382.

Poleski, J. 2013: Małopolska w VI-X wieku. Studium archeologiczne. Kraków: Towarzystwo Wydawnicze "Historia lagellonica".

Pollak, R. 2017: The early Islamic green lead glass from the excavations at Caesarea Maritima, Israel. In: S. Wolf - A. de Pury-Gysel eds., Annales du $20^{\circ}$ Congrès de l'Association Internationale pour I'Histoire du Verre. Fribourg/Romont 7-11 septembre, Remont: Verlag Marie Leidorf, 288-292.

Procházka, R. 2018: Přerov stronghold and the material culture of its inhabitants in the late phase of the Early Middle Ages. In: P. Kouriil - R. Procházka eds., Moravian and Silesian Strongholds of the Tenth and Eleventh Centuries in the Context of Central Europe, Brno: Archeologický ústav AV ČR, 137-170. 
Ratajczyk, Z. 2013: The cemetery in Ciepłe - current research results. In: S. Moździoch et al. eds., Scandinavian culture in medieval Poland, Wrocław: Instytut Archeologii i Etnologii PAN, 323-347.

Rębkowski, M. 2019: Central settlement of medieval Wolin, In: M. Rębkowski ed., Wolin. The Old Town. Vol. I. Settlement Structure, Stratigraphy \& Chronology, Szczecin: Instytut Archeologii i Etnologii PAN, 133-150.

Robertshaw, P. - Benco, N. - Wood, M. - Dussubieux, L. - Melchiorre, E. - Ettahiri, A. 2010: Chemical analysis of glass beads from medieval Al-Basra (Morocco). Archaeometry 52, 355-379.

Romanow, J. - Wachowski, K. - Miszkiewicz, B. 1973: Tomice, pow. Dzierżoniów. Wielokulturowe stanowisko archeologiczne, Wrocław: Zakład Narodowy im. Ossolińskich.

Romańska, A. 2014a: III. Okres wpływów rzymskich. In: P. Bobrowski ed., Badania archeologiczne na terenie „Zbiornika przeciwpowodziowego Racibórz Dolny na rzece Odrze, województwo śląskie (polder)". Tom III/2. Tworków, stanowisko 9 (ZRD 9), III sezon badań, rok 2014, Poznań - Wrocław: Instytut Archeologii i Etnologii PAN, etc., 51-80.

Romańska, A. 2014b: IV. Okres wpływów rzymskich. In: D. Minta-Tworzowska ed., Badania archeologiczne na terenie „Zbiornika przeciwpowodziowego Racibórz Dolny na rzece Odrze, województwo śląskie (polder)". Tom III/1. Tworków, stanowisko 9 (ZRD 9), Poznań - Wrocław: Instytut Archeologii i Etnologii PAN, etc., 109-232.

Schejbalová, Z. 2011: Raně středověká řadová pohřebiště v Plzeňském kraji. Plzeň: Petr Mikota.

Sedláčková, H. - Rohanová, D. et al. 2016: Renaissance and Baroque Glass from the Central Danube Region. Brno: Archaia Brno.

Siegmann, M. 2007: Kleinst - Glas - Miniatur - Stick - Perl - Collier oder: Form ist nicht Funktion. Miniaturperlen im archäologischen Befund. In: F. M. Andraschko ed., Archäologie zwischen Befund und Rekonstruktion. Festschrift Renate Rolle. Antiquitates 39, Hamburg: Kovač, 275-289.

Siegmann, M. 2009: Landpomeranzen oder voll im Trend? Schmuck und Modebewusstsein der Frauen von Liebenau, Kr. Nienburg/Weser und Dörverden, Kr. Verden. In: O. Heinrich-Tamaska et al. eds., Dunkle Jahrhunderte in Mitteleuropa? Tagungsbeiträge der Arbeitsgemeinschaft Spätantike und Frühmittelalter, Hamburg: Kovač, 135-158.

Siemianowska, E. 2008: Wczesnośredniowieczne grzechotki i pisanki w strefie przebiegu szlaku lądowego z Rusi na Pomorze. In: P. Kucypera - S. Wadyl eds., Kultura materialna średniowiecza w Polsce, Toruń: Wydawnictwo Naukowe Uniwersytetu Mikołaja Kopernika, 67-84.

Siemianowska, S. 2017: Domniemana karczma i "dom kupca” na śląskim grodzie. Socjotopografia wczesnośredniowiecznego Opola-Ostrówka w świetle znalezisk szklanych i przedmiotów towarzyszących. In: D. Adamska et al. eds., Cum gratia et amicitia. Studia z dziejów osadnictwa dedykowane Pani Profesor Marcie Młynarskiej-Kaletynowej z okazji 65-lecia działalności naukowej, Wrocław: Instytut Archeologii i Etnologii PAN - Instytut Archeologii Uniwersytetu Wrocławskiego, 151-158.

Siemianowska, S. 2020: Przemiany społeczno-gospodarcze doby przełomu lokacyjnego na Śląsku. Szkło jako identyfikator zmian. In: K. Chrzan et al. eds., Ceramika i szkło w badaniach interdyscyplinarnych, Wrocław: Instytut Archeologii i Etnologii PAN, 195-228.

Siemianowska, S. in print: Glass gaming pieces for a board game from Opole-Ostrówek - Was hnefatafl played in the early medieval stronghold?. In: Interdisciplinary Medieval Studies IV. Yeast for Changes, Wrocław: Instytut Archeologii i Etnologii PAN.

Siemianowska, S. - Pankiewicz, A. - Sadowski, K. 2019: On technology and production techniques of early medieval glass rings from Silesia. Archaeometry 61, 614-646.

Siemianowska, S. - Pankiewicz, A. - Sadowski, K. in print: W kwestii techniki wykonania i szkliwienia wczesnośredniowiecznych pisanek-grzechotek ze Śląska. Silesia Antiqua 52.

Siemianowska, S. - Sadowski, K. 2019: Późnośredniowieczne naczynia szklane z Legnicy w świetle badań interdyscyplinarnych. Śląskie Sprawozdania Archeologiczne 61, 147-175.

Skomsvoll, J. 2012: Nordnorske spillsaker fra jernalder og middelalder (PDF). https://munin.uit.no/handle/ 10037/4310

Sláma, J. 1977: Mittelböhmen im frühen Mittelalter. I. Katalog der Grabfunde. Praehistorica V. Praha: Univerzita Karlova.

Stanistawski, B. 2013: Norse culture in Wolin-Jómsborg. In: S. Moździoch et al. eds., Scandinavian culture in medieval Poland, Wrocław: Instytut Archeologii i Etnologii PAN, 193-241.

Stǎšíková-Štukovská, D. - Ungerman, Š. 2009: Sklené korálky z včasnostredovekého pohrebiska v Dolních Věstoniciach, In: P. Dresler - Z. Měřínský eds., Archeologie doby hradištní v České a Slovenské republice, Archaeologia mediaevalis Moravica et Silesiana - Suppl. 2, Brno: Masarykova univerzita, 136-149. 
Steppuhn, P. 1998: Die Glasfunde von Haithabu, Berichte über die Ausgrabungen in Haithabu 32. Neumünster: Wachholtz.

Ślusarski, K. W. 2004: Wczesnośredniowieczne pisanki i grzechotki z ziem polskich. Próba typologii. In: Z. Kobyliński ed., Hereditatem cognoscere. Studia i szkice dedykowane Profesor Marii Miśkiewicz, Warszawa: Państwowe Muzeum Archeologiczne, 79-123.

Tempelmann-Mq̨czyńska, M. 1985: Die Perlen der römischen Kaiserzeit und der frühen Phase der Völkerwanderungszeit im mitteleuropäischen Barbaricum. Mainz: Philipp von Zabern.

Ungerman, Š. 2007: Raně středověké pohřebiště v Dolních Věstonicích - Na pískách. Manuscript in the archiv of the Filozofická fakulta, Ústav archeologie a muzeologie, Masarykova univerzita, Brno.

Wachowski, K. 1975: Cmentarzyska doby wczesnopiastowskiej na Śląsku. Wrocław etc.: Zakład Narodowy im. Ossolińskich.

Wachowski, K. 1984: Militaria z grodu na Ostrówku w Opolu. In: B. Gediga ed., Studia nad kulturą wczesnopolskiego Opola. Militaria - wyroby bursztynowe, Wrocław: Zakład Narodowy im. Ossolińskich, 11-112.

Wachowski, K. 2001: Elementy rodzime i obce w uzbrojeniu wczesnośredniowiecznym na Śląsku. Acta Universitatis Lodziensis. Folia Archaeologica 23, 153-176.

Wachowski, K. - Domański, G. 1992: Wczesnopolskie cmentarzysko w Starym Zamku. Wrocław: Oficyna Wydawnicza Sudety.

Wajda, S. 2013: Zabytki szklane i szkliwione. In: J. Kalaga ed., Sutiejsk. Gród pogranicza polsko-ruskiego zX-XIII wieku. Studium interdyscyplinarne, Warszawa - Pękowice: Instytut Archeologii Uniwersytetu Warszawskiego, 89-100.

Wajda, S. 2019: Paciorki szklane. In: S. Wadyl ed., Ciepłe. Elitarna nekropola wczesnośredniowieczna na Pomorzu Wschodnim, Gdańsk: Muzeum Archeologiczne w Gdańsku, 216-231.

Wedepohl, K. H. - Krueger, I. - Hartman, G. 1995: Medieval lead glass from northwestern Europe. Journal of Glass Studies 37, 65-66.

Wołoszyn, M. 2004: Zabytki pochodzenia wschodniego we wczesnośredniowiecznej Polsce - wędrówka ludzi, rzeczy, czy idei?. In: S. Moździoch ed., Wędrówki rzeczy i idei w średniowieczu. Spotkania Bytomskie 5, Wrocław: Instytut Archeologii i Etnologii PAN, 241-259.

ALEKSANDRA PANKIEWICZ, Institute of Archaeology, University of Wrocław, Szewska 48, PL-50-139 Wrocław ohlap@poczta.onet.pl SYLWIA SIEMIANOWSKA, Institute of Archaeology and Ethnology of the Polish Academy of Sciences, Centre for Late Antique and Early Medieval Studies, Więzienna 6, PL-50-118 Wrocław; sylwiasiemianowska@wp.pl 